\title{
Topological phase-fluctuations, amplitude fluctuations, and criticality in extreme type-II superconductors
}

\author{
A. K. Nguyen ${ }^{1}$ and A. Sudb $\varnothing^{1,2}$ \\ ${ }^{1}$ Norwegian University of Science and Technology, N-7034 Trondheim, Norway \\ ${ }^{2}$ California Institute of Technology, Pasadena, CA91125, USA
}

\begin{abstract}
We study the effect of critical fluctuations on the $(B, T)$ phase diagram in extreme type-II superconductors in zero and finite magnetic field. In zero magnetic field the critical fluctuations are transverse phase-fluctuations of the complex scalar Ginzurg-Landau order parameter, which when excited thermally will induce line-defects in the form of closed vortex loops into the system. The distribution function $D(p)$ of vortex loops of perimeter $p$ changes from an exponential function $D(p) \sim p^{-\alpha} \exp \left(-\varepsilon(T) p / k_{B} T\right)$ to a power law distribution $D(p) \sim p^{-\alpha}$ at the zero-field critical temperature $T=T_{c}$. We find that the long-wavelength vortex-line tension vanishes as $\varepsilon(T) \sim$ $\left|T-T_{c}\right|^{\gamma} ; \gamma \approx 1.45$, as $T \rightarrow T_{c}$. At $T=T_{c}$, an extreme type-II superconductor suffers an unbinding of large vortex loops of order the system size. When this happens, the connectivity of the thermally excited vortex-tangle of the system changes abruptly. When amplitude fluctuations are included, it is shown that they are far from being critical at the superconducting transition temperature $T_{c}$. The vortex-loop unbinding can therefore not be reparametrized in terms of critical amplitude fluctuations of the original local Ginzburg-Landau order parameter. The loss of phase-stiffness in the GinzburgLandau order parameter, the anomaly in specific heat, the loss of vortex-line tension, and the change in the connectivity of the vortex-tangle are all found at the same temperature, the critical temperature of the superconductor. At zero magnetic field, unbinding of vortex-loops of order the system size can be phrased in terms of a global $U(1)$-symmetry breaking involving a local complex disorder field which is dual to the order parameter of the usual Ginzburg-Landau theory. There is one parameter in the theory that controls the width of the critical region, and for the parameters we have used, we show that a vortex-loop unbinding gives a correct picture of the zero-field transition even in the presence of amplitude fluctuations. A key result is the extraction of the anomalous dimension of the dual field directly from the statistics of the vortex-loop excitations of the GinzburgLandau theory in the phase-only approximation. In finite magnetic fields, the first order vortex-line lattice (VLL) melting transition is accompanied by a loss of longitudinal superfluid stiffness; this is true also for the isotropic case. A scaling analysis of the vortex lattice melting line is carried out, yielding two different scaling regimes for the vortex lattice melting line, namely a high-field scaling regime and a distinct low-field $3 D X Y$ scaling regime. We also find indications of an abrupt change in the connectivity of the vortex-tangle in the vortex liquid along a line $T_{L}(B)$, which at low enough fields appears to coincide with the VLL melting transition line within the resolution of our numerical calculations. We study the temperature at which this phenomenon takes place as a function of system size and shape. Our results show that this temperature decreases and appears to saturate with increasing system size, and is insensitive to aspect ratios of the systems on which the simulations are performed on, for large enough systems. A necessary, but not sufficient, condition for the vortex-line tension to vanish is a change in the connectivity of the vortex tangle in an extreme type-II superconductor.
\end{abstract}

Pacs-numbers: 74.20.De, 74.25.Dw, 74.25.Ha,74.60.Ec

\section{INTRODUCTION}

Ten years after Abrikosov's classic prediction of a lattice of quantized vortices, the Abrikosov vortex-line lattice (VLL), as the ground state of type-II superconductors when the magnetic field is tuned beyond a lower critical value 2 , Gerd Eilenberger suggested that the VLL could melt close to the critical temperature of the system 3 . The magnetic field versus temperature $(B, T)$ phase diagram of extreme type-II superconductors has for some time been under intense investigation both theoretically and experimentally, following suggestions that the VLL could undergo a melting transition in regime of the $(B, T)$-phase diagram of the high-temperature $\mathrm{pt}$ perconductor that could be experimentally resolved 6 .
This was soon confirmed by a more thorough theoretical analysis 9 where it was shown that the VLL of the hightemperature superconductors was particularly susceptible to thermal fluctuations due to the large anisotropy of these compounds. The anisotropy only affects the melting line of the VLL when the pronounced nonlocal elastic properties of the VLL in strong type-II superconductors, first discussef for the isotropic case in the pioneering works of Brandt 6 , are taken into account 6 .

While it now appears well established both experimentally 9 and theoretically for three dimensional vortex systems 10.11 that the VLL in the clean limit of type-II superconductors melts in a first order phasetransition, much less consensus has been reached on how to describe the state which the VLL melts into, even 
in the clean limit. Only very recently has if been established, through numerical simulations 1213 that the vortex-liquid is always incoherent, i.e phase-coherence is destroyed in all direction, including the direction of the induction, as soon as the VLL melts. Inside the vortexliquid regime, there is therefore no transition from a disentangled to an entangled vortex-liqiud. For such a transition to occur inside the vortex-liquid, the longitudinal superfluid density would have to be non-zero above the melting temperature This however does not happen in the clean limit 12.13, even in the isotropic case 14 . Recently, questions have also been raised whether the vortex-line picture of the molten phase of the Abrikgsov VLL is viable at all at low fields $B<17$ 15 17,23 . 18 . 14 .

In terms of fundamental physics, extreme type-II superconductors are interesting due to their large fluctuation effects not commonly seen in condensed matter systems. This is ultimately due to the fact that they are strong coupling superconductors arising out of doped Mott-Hubbard insulators. The latter fact gives rise to the effect that the phase-stiffness of the superconducting order parameter is low, due to a low value of the superfluid density $\rho_{s}$

$$
\rho_{s} \sim \frac{\partial^{2} f}{\partial(\Delta \theta)^{2}},
$$

where $\Delta \theta$ is a twist in the superconducting order parameter over the size of the system, and $f$ is the free energy density. This particular and important aspect of doped Mott-Hubbard insulators has - pen quite strongly emphasized already for some time 29 21, see also Ref. 22. The strong coupling effect gives rise to a large $T_{c}$, so that the Ginzburg-Landau parameter $\kappa \sim T_{c} / \sqrt{\rho_{s}}$ is large. This also softens the vortex matter in these systems, farticularly when coupled with their strong layeredness 9 .

There is also a close connection between thermodynamic phase transitions in these system and phasetransitions in superfluids23, liquid crystals 24 , crystal 25 , and cosmology 26 30 . The close connection between these apparently different physical problems, is due to the similarity of the topological objects that appear in these problems. Particularly in the context of superfluid $\mathrm{He}^{4}$, the proposition that an unbinding of topological phasefluctuations in the form of vortex-loops is the microscopic mechanism for the superfluid-normal state transition, has been extensively studied in the past 3135,36 , and early attempts at formulating a field-theory of this in the context of charged superfluids in zero magnetic field has also appeared in the literature 37 . Effective gauge-field theories with an internal $U(1)$-symmetry all have in common that they support line-defect in the form of vortex-loop excitations as stable topological objects. Understanding the role of such excitations on the $(B, T)$ phase-diagram of type-II superconductors is an important problem in physics, and presumably will shed light on the related problems mentioned above as well.

In conventional low-temperature superconductors, the temperature where Cooper pairs start to form, $T_{M F}$, is practically identical to the true superconducting transition temperature $T_{c}$. The commonly applied Ginzburgcriterion provides a useful estimate for the width of the critical regions in systems with weak fluctuation effects, showing that the width of the critical region is of order $|t| \sim\left(T_{c}-T\right) / T_{c} \sim 10^{-6}-10^{-4}$. A mean field description of the S-N phase transition is appropriate 'for all practical purposes". In high- $T_{c}$ superconductors, this may no longer be the case. There appears to be mounting experimental evidence that the width of the critical region is as large as a few Kelvin in $\mathrm{YBCO}_{3}$ 42 2 , which would encompass the melting line of the flux-line lattice up to a field of order $1 T$ l.

In zero field the superconducting-normal phase transition is exclusively caused by a vortex loop unbinding $17,43.14,44,45$. Below the critical temperature $T_{c}$ vortex loops are confined to a typical perimeter $L_{0}$, and cause only local disturbances in the macroscopic superconducting state. Recently, this has been demonstrated clearly, by correlating an abrupt change in vortex tangle connectivity, a loss of vortex-line tension, loss of superfluid stiffness and specific heat anomaly precisely at the critical temperature of the superconductor, even for the isotropic case 43.44. At $T_{c}$, thermally induced vortex loops loose their effective line tension and therefore unbind.

In this scenario, at low fields, thermally induced vortex loops could conceivably interact strongly with field induced flux lines. This interaction is ignored in models using the line-only approximation, where the thermally induced vortex loops degrees of freedom are neglected, effectively being considered as irrelevant relativistic corrections in a corresponding $2 D$ quantum boson system.146.47. This model has met with considerable success in describing parts of the flux-line lattice melting curve at intermediate to elevated fields, where its position the phase-diagram as well as its dependence on anisgtropy was explained using the Lindemanncriterion 148 46. The melting line for fields of more than a few Tesla is little affected by the vortex-loop unbinding, as pointed out recently 43.4 .

However, the question arises whether this is a tenable conclusion for low fields as well. The fact that the zerofield transition can be characterized precisely by a loss of line-tension of thermally induced flux lines, implies that there is a sharp change in the distribution function $D(p)$ for vortex loops of a given perimeter $p$. It changes from an exponential form $D(p) \sim p^{-5 / 2} \exp \left(-\varepsilon(T) p / k_{B} T\right)$ to a power law $D(p) \sim p^{-5 / 2}$ at $T_{c} 14$. This implies the existence of a diverging length scale $L_{0}(T)=k_{B} T / \varepsilon(T) 43$. 44 . Given this fact, it raises the question of whether the critical fluctuations can affect the melting line in a sizeable field-temperature regime, rendering the vortex lines tension-less. The vortex line tension is analagous to the mass of the bosons in a $2 D$ non-relativistic boson-analogy of the vortex system. If the vortex-line tension were to vanish, it would mean that the boson-mass wouild vanish in the corresponding analogy. There is no non-relativistic 
limit of any mass-less theory. The conclusion would be that any $2 D$ boson-model which is non-relativistic, is inapplicable in the part of the phase-diagram where the vortex-line tension vanishes. We reemphasize that at elevated fields, where the first order flux-line lattice melting line splits off from the new transition line proposed here, the Lindemann-criterion of flux-line lattice melting is expected to correctly locate the position of the melting line 43 .

The outline of this paper is as follows. In Section II, we introduce the Ginzburg-Landau model studied in this paper, and various approximations and reformulations of it, as well as their inter-relations. In Section III we present the ideas underlying the simulations that are presented in this paper, and introduce and discuss the quantities we study. In Section IV, we present results of the simulations in zero magnetic field. In particular, we present results which demonstrate that the zero-field transition in an extreme type-II superconductor is driven by a proliferation of unbound vortex loops, which therefore constitute the critical fluctuations of this system. In Section V, finite-field results are given. Summary and conclusions are presented in Section VI, and in this section we also list point by point the new results obtained in this paper.

\section{MODELS}

In this section we define the models considered in this paper: 1) the continuum Ginzburg-Landau model, 2) the lattice Ginzburg-Landau model in a frozen gauge approximation, and 3) the uniformly frustrated 3D XY model. We also discuss the approximations involved and the validity of the models.

\section{A. Ginzburg-Landau model}

Our starting point is the continuum Ginzburg-Landau (GL) model50. In quantum field theory, the GL model is also referred as the scalar QED or the $U(1)+$ Higgs model or the Abelian Higgs model. The effective Hamiltonian for the GL model in an anisotropic system is given by 51

$$
\begin{aligned}
H_{G L} & =\int d^{3} r\left[\alpha(T)|\psi|^{2}+\frac{g}{2}|\psi|^{4}\right. \\
& +\sum_{\mu=x, y, z} \frac{\hbar^{2}}{2 m_{\mu}}\left|\left(\nabla_{\mu}-i \frac{2 \pi}{\Phi_{0}} A_{\mu}\right) \psi\right|^{2} \\
& \left.+\frac{1}{2 \mu_{0}}(\nabla \times \mathbf{A})^{2}\right] .
\end{aligned}
$$

Here, $\psi(\mathbf{r})=|\psi(\mathbf{r})| e^{i \theta(\mathbf{r})}$ is a complex order field representing the superconducting condensate. In superconductors, the amplitude $|\psi(\mathbf{r})|^{2}$ should, be interpreted as the local Cooper-pair density. Furthermore, $m_{\mu}$ is the effective mass for one Cooper pair when moving along the $\mu$-direction, $\Phi_{0}=h / 2 e$ is the flux quantum, and $\mu_{0}$ is the vacuum permeability. In Eq. 1, the gauge field $\mathbf{A}$ is related to the local magnetic induction, $b(\mathbf{r})=\nabla \times \mathbf{A}(\mathbf{r})$. Finally, the GL parameter $g$ is assumed to be temperature independent, while $\alpha=\alpha(T)$ changes sign at a mean field critical temperature $T_{M F}(B)$, where Cooper pairs start to form. $B$ is the spatial average of the magnetic induction. The critical temperature $T_{c}$ where phase-coherence develops, is always smaller than $T_{M F}$; the existence a finite Copper-pair density does not imply that the system is in a superconducting state.

Later on, we shall recast the Ginzburg-Landau theory in a quite different form that also exhibts a $U(1)$ symmetry, but where the field conjugate to the relevant phase is the number operator for the topological excitations destroying the order of the Ginzburg-Landau theory itself. Although this may seem like an unnecessary complication, it has the advantage of facilitating a detailed discussion of the vortex-liquid phase of the GL-theory in terms of the ordering of some local field, namely the complex scalar field $\phi(\mathbf{r})$ to be introduced and discussed in Section IIF. This is not possible using the GinzburgLanday function, $\psi(\mathbf{r})$, since $\psi(\mathbf{r})>$ is always zero in the vortex liquid phase 2 213. In the zero-field lowtemperature ordered phase, the system spontaneously chooses a preferred phase angle $\Theta$, and explicitly breaks the $U(1)$ symmetry. The vortex-sector of the GL-theory also exhibits a $U(1)$-symmetry breaking, but where $U(1)$ symmetry is broken in the high-temperature phase, and restored in the low-temperature phase.

Eq. 11 has two intrinsic length scales, the mean-field coherence length

$$
\xi_{\mu}^{2}(T)=\frac{\hbar^{2}}{2 m_{\mu}|\alpha(T)|},
$$

and the magnetic penetration depth

$$
\lambda_{\mu}^{2}=\frac{m_{\mu} \beta}{4 \mu_{0} e^{2}|\alpha(T)|} .
$$

$\xi_{\mu}$ is the characteristic length of the variation of $|\psi(\mathbf{r})|$ along the $\mu$-direction, and $\lambda_{\mu}$ is the characteristic length of the variation of the current flowing along the $\mu$ direction.

In order to carry out Monte Carlo simulations of the GL model, the model is discretized by replacing the covariant derivative in the continuum GL Hamiltonian, Eq. 11, with a covariant lattice derivative,

$$
\begin{aligned}
D_{\mu} \psi & =\left(\nabla_{\mu}-i \frac{2 \pi}{\Phi_{0}} A_{\mu}\right) \psi \\
\rightarrow D_{\mu} \psi & =\frac{1}{a_{\mu}}\left(\psi(\mathbf{r}+\hat{\mu}) e^{-i \frac{2 \pi}{\Phi_{0}} a_{\mu} A_{\mu}(\mathbf{r})}-\psi(\mathbf{r})\right) .
\end{aligned}
$$

The resulting model is a version of the Lawrence-Doniach mode 52 with all three directions discretized. The effective Hamiltonian for the lattice GL model is given by, 


$$
\begin{aligned}
H_{L G L} & =a_{x} a_{y} a_{z} \sum_{\mathbf{r}}\left[\alpha|\psi|^{2}+\frac{g}{2}|\psi|^{4}\right. \\
& +\sum_{\mu=x, y, z} \frac{\hbar^{2}}{2 m_{\mu} a_{\mu}^{2}}\left|\psi(\mathbf{r}+\hat{\mu}) e^{-i \frac{2 \pi}{\Phi_{0}} a_{\mu} A_{\mu}(\mathbf{r})}-\psi(\mathbf{r})\right|^{2} \\
& \left.+\sum_{\mu=x, y, z} \frac{1}{2 \mu_{0} a_{\mu}^{2}}(\boldsymbol{\Delta} \times \mathbf{A})_{\mu}^{2}\right] .
\end{aligned}
$$

Here, $a_{\mu}$ and $\hat{\mu}$ is a lattice constant and a unit vector along the $\mu$-axis, respectively. Furthermore, the lattice derivative is defined as

$$
\Delta_{\mu} \psi(\mathbf{r})=\psi(\mathbf{r}+\hat{\mu})-\psi(\mathbf{r}) .
$$

Taking the continuum limit $a_{\mu} \rightarrow 0$, the effective Hamiltonian for the lattice GL model (Eq. 5) reduces correctly to the GL effective Hamiltonian in the continuum (Eq. 11). As defined in Eq. 5, the lattice GL model does not contain vortices. To reintroduce the vortices in the model, we must compactify the gauge-theory by requiring that the gauge invariant phase differences satisfy 53 ,

$$
\left[\theta(\mathbf{x}+\hat{\mu})-\theta(\mathbf{x})-\frac{2 \pi}{\Phi_{0}} a_{\mu} A_{\mu}(\mathbf{x})\right] \in[-\pi, \pi>.
$$

Whenever this constraint is used to bring the gauge invariant phase differences back to its primary interval, we automaticall introduce a unit closed vortex loop, and the net vorticity of the system is guaranteed to be conserved at every stage of the Monte-Carlo simulation. From the renormalization group point of view the continuum GL model and the lattice GL model belong to the same universality class 25. We therefore expect the lattice GL model and the continuum GL model to give, qualitatively, the same results.

\section{B. Lattice Ginzburg-Landau model in a frozen gauge approximation}

In extreme type-II superconductors, the zero temperature mean-field penetration depth is much greater than the zero temperature coherence length, $\lambda_{\mu}(T=0) \gg$ $\xi_{\mu}(T=0)$. Thus, fluctuations of the gauge field represented by the last term in Eq. \& around the extremal field configuration are strongly suppressed and can therefore be neglected. The effective Hamiltonian for the frozen gauge (FG) model can be written as

$$
\begin{aligned}
H_{F G} & =\frac{|\alpha(0)|^{2}}{g} a_{x} a_{y} a_{z} \sum_{\mathbf{r}}\left[\frac{\alpha(T)}{\alpha(0)}\left|\psi^{\prime}\right|^{2}+\frac{1}{2}\left|\psi^{\prime}\right|^{4}\right. \\
& \left.+\sum_{\mu=x, y, z} \frac{\xi_{\mu}^{2}}{a_{\mu}^{2}}\left|\psi^{\prime}(\mathbf{r}+\hat{\mu})\right|\left|\psi^{\prime}(\mathbf{r})\right|\left[2-2 \cos \left(\Delta_{\mu} \theta-\mathcal{A}_{\mu}\right)\right]\right] .
\end{aligned}
$$

Here, we have defined a dimensionless order field and vector potential

$$
\begin{gathered}
\psi^{\prime}=\frac{\psi}{\sqrt{\frac{|\alpha(0)|}{g}}} \rightarrow\left|\psi^{\prime}\right| \sim[0,1], \\
\mathcal{A}_{\mu}=\frac{2 \pi}{\Phi_{0}} a_{\mu} A_{\mu} .
\end{gathered}
$$

The natural energy scale along the $\mu$-direction is,

$$
J_{\mu}=2 \frac{|\alpha(0)|^{2}}{g} a_{x} a_{y} a_{z} \frac{\xi_{\mu}^{2}}{a_{\mu}^{2}} .
$$

Assuming a uniaxial anisotropy along the $z$-axis, the natural energy scale for the FG model is

$$
J_{0}=J_{x}=\frac{2|\alpha(0)|^{2}}{g} \xi_{a b}^{2} a_{z}=\frac{\Phi_{0}^{2} d}{4 \pi^{2} \mu_{0} \lambda_{a b}^{2}} .
$$

Here, we have put our coordinates $(x, y, z)$-axis parallel to the crystals $(a, b, c)$-axis. Furthermore, $\xi_{x}=\xi_{y}=\xi_{a b}$ and $\xi_{z}=\xi_{c}$ is the coherence length in the $\mathrm{CuO}$-planes and along the crystal's $c$-axis, respectively. Furthermore, $\lambda_{x}=\lambda_{y}=\lambda_{a b}$ and $\lambda_{z}=\lambda_{c}$ is the penetration depth in the $\mathrm{CuO}$-planes and along the crystals $c$-axis, respectively. In Eq.8, $d$ is the distance between two $\mathrm{CuO}$ superconducting planes in adjacent unit cells. The energy scale $J_{0}$ is reurhly the energy scale of exciting a unit vortex loop 19.54 .13$.

The ratio between the energy scales $J_{x}$ and $J_{z}$ serves as an anisotropy parameter,

$$
\Gamma=\sqrt{\frac{J_{x}}{J_{z}}}=\frac{\xi_{a b} a_{z}}{\xi_{c} a_{x}}=\frac{\lambda_{c} a_{z}}{\lambda_{a b} a_{x}} .
$$

In this model, the lattice constant $a_{\mu}$ should be defined as

$$
a_{\mu}=\max \left(\mathrm{d}_{\mu}, \mathrm{C}_{0} \xi_{\mu}\right) .
$$

Here, $d_{\mu}$ is an intrinsic length along the $\mu$-direction in the underlying superconductor to be modeled. Examples of such intrinsic length are the distance between $\mathrm{CuO}$ planes in adjacent unit cells, the (a,b)-dimension of the unit cell. To be consistent, the constant $C_{0}$ should be larger than $\sim 4$. This requirement $a_{\mu} / \xi_{\mu}>4$ ensures that the amplitude of the order field does not overlap55. Such overlap will give rise to a domain wall term $(\nabla|\psi|)$, which is absent in the lattice GL model.

Within the frozen gauge approximation, the gauge field serves only as a constraint in fixing the value of the uniform induction. In terms of magnetic induction this approximation is valid when $B \gg B_{c 1}(T)$, where the field distribution from individual flux lines overlap strongly, giving uniform induction. Note that $B_{c 1}(T)$ also vanishes when the temperature approaches $T_{c}$. In zero field, this approximation is valid for all temperatures except an inaccessible temperature region around $T 56$.

In our simulations on the FG model, we allow for both phase- and amplitude fluctuations of the superconducting order parameter $\psi(\mathbf{r})=|\psi(\mathbf{r})| \exp [i \theta(\mathbf{r})]$. Details of the Monte-Carlo procedure for this case will be given below. 


\section{Uniformly frustrated 3D XY model}

The uniformly frustrated 3D XY model was first used as a phenomenological model for extreme ype-II superconductors by Li et al.54 and Hetzel et al.11. To obtain the uniformly frustrated 3D XY (3DXY) model from the FG model, we freeze the amplitude of the complex order field in Eq. 7, $\left|\psi^{\prime}\right|=1$. This is the London approximation. The resulting effective Hamiltonian for the 3DXY model is given by

$$
H_{X Y}=-\frac{2|\alpha(0)|^{2}}{g} a_{x} a_{y} a_{z} \sum_{\mathbf{r}, \mu} \frac{\xi_{\mu}^{2}}{a_{\mu}^{2}} \cos \left(\Delta_{\mu} \theta-\mathcal{A}_{\mu}\right)
$$

The lattice constants in the 3DXY model should be defined as

$$
a_{\mu}=\max \left(\mathrm{d}_{\mu}, \xi_{\mu}\right)
$$

Assuming uniaxial anisotropy, the energy scales and the anisotropy parameter of the 3DXY model are the same as for the FG model, Eqs. 8, 9. Note that both the FG model and the 3DXY model contain precisely the same topological objects, i.e. vortex loops and vortex lines, as for the GL model. The local gauge symmetry in the GL model is however reduced to a global $U(1)$-symmetry in the FG model and the 3DXY model.

\section{Villain-approximation and vortex representation}

To further corroborate interpretations of the results from our Monte-Carlo simulations using the uniformly frustrated $3 D X Y$-model, to be detailed in the next section, it is useful to provide an alternative, but entirely equivalent formulation of the GL-theory. This formulation replaces a description in terms of the GL-function $\psi$ by vortex-degrees of freedom, where the interaction between vortex-segments is mediated by a gauge-field, which we denote by $\mathbf{h}$. This gauge-field is not the electromagnetic vector potential $\mathbf{A}$, but will couple to it. The resulting structure of the theory makes it possible, in three dimensions, and three dimensions only, to reformulate the vortex-content of the GL-theory as a theory of a complex matter field $\phi$ coupled to the gauge-fields $\mathbf{h}$ and A. Although this may seem as an unnecessary detour, the great advantage of this approach, is that certain vortex-correlators, notably our quantity $O_{L}$ to be defined below, can be directly related to a $U(1)$-symmetry of the $\phi$-theory.

To proceed with this, we introduce the well-known Villain-approximation to the 3DXY model. The Villain approximation consists of replacing the cosine potential in the uniformly frustrated $3 D X Y$-model by a Gaussian $2 \pi$-periodic potential. In this way the longitudinal spinwave excitations of the $\theta$-field decouple from transverse vortex-excitations of the theory. This decoupling does not alter the critical behavior of the system. The partition function for this theory reads, after a rescaling of the vector potential and charge

$$
\begin{aligned}
Z_{V} & =\prod_{\mathbf{r}} \int D \theta(\mathbf{r}) \int D A(\mathbf{r}) \sum_{\mathbf{n}(\mathbf{r})=-\infty}^{\infty} e^{S} \\
S & =-\sum_{\mathbf{r}}\left[\frac{\beta}{2}(\nabla \theta-2 e \mathbf{A}-2 \pi \mathbf{n})^{2}+\frac{1}{2}(\nabla \times \mathbf{A})^{2}\right],
\end{aligned}
$$

where $\mathbf{n}(\mathbf{r})$ is an integer-valued field. The kinetic term is linearized by a Hubbard-Stratonovich decoupling, introducing an auxiliary velocity-field $\mathbf{v}(\mathbf{r})$ and using the identity

$$
\prod_{\mathbf{r}} e^{-\beta u^{2}(\mathbf{r}) / 2} \sim \prod_{\mathbf{r}} \int D \mathbf{v} \exp \left(-\sum_{\mathbf{r}}\left[\frac{\mathbf{v}(\mathbf{r})^{2}}{2 \beta}-i \mathbf{v}(\mathbf{r}) \cdot \mathbf{u}(\mathbf{r})\right]\right)
$$

This is now inserted back into the original partition function, using $\mathbf{u}=\nabla \theta-2 e \mathbf{A}-2 \pi \mathbf{n}(\mathbf{r})$. The sum over the integers $\mathbf{n}(\mathbf{r})$ may be carried out, yielding the constraint that $\mathbf{v}(\mathbf{r})$ is integer valued, say $\mathbf{v}(\mathbf{r})=\mathbf{l}(\mathbf{r})$. The next step is to integrate out the $\theta(\mathbf{r})$-variable, which yields the constraint $\nabla \cdot \mathbf{v}(\mathbf{r})=0$, which is solved by introducing an integer valued field $\mathbf{h}(\mathbf{r})$ such that $\mathbf{l}(\mathbf{r})=\nabla \times \mathbf{h}(\mathbf{r})$. In order to be able to treat $\mathbf{h}$ as a continuous variable, we introduce a new integer-valued field $\mathbf{m}$ and apply the Poisson-summation formula

$$
\sum_{\mathbf{m}=-\infty}^{\mathbf{m}=\infty} e^{2 \pi i \mathbf{m} \cdot \mathbf{h}}=\sum_{\mathbf{l}=-\infty}^{\infty} \delta(\mathbf{l}-\mathbf{h})
$$

Note that this procedure does not involve any approximations. Finally, we write the partition function for the GL-theory in phase-only and Villain-approximations as

$$
\begin{aligned}
Z & =\prod_{\mathbf{r}} \int D \mathbf{h} D \mathbf{A} \sum_{\mathbf{m}=-\infty}^{\infty} e^{S_{\mathrm{eff}}[\mathbf{m}, \mathbf{h}, \mathbf{A}]} \\
S_{\mathrm{eff}} & =-\sum_{\mathbf{r}}\left[2 \pi i \mathbf{m} \cdot \mathbf{h}+\frac{1}{2 \beta}(\nabla \times \mathbf{h})^{2}+2 i e(\nabla \times \mathbf{h}) \cdot \mathbf{A}\right. \\
& \left.+\frac{1}{2}(\nabla \times \mathbf{A})^{2}\right]
\end{aligned}
$$

where the following constraints apply in the functional integral: $\nabla \cdot \mathbf{A}=\nabla \cdot \mathbf{h}=\nabla \cdot \mathbf{m}=0$. The effective action, Eq. 11, is invariant under

$$
\begin{aligned}
& \mathbf{h} \rightarrow \mathbf{h}+\nabla \omega_{\mathrm{h}} \\
& \mathbf{A} \rightarrow \mathbf{A}+\nabla \omega_{\mathrm{A}} .
\end{aligned}
$$

The field $\mathbf{h}$ is readily interpreted as a fictitious gauge-field that mediates an interaction between vortex-segments $\mathbf{m}$. This is easily seen by integrating out the $\mathbf{h}$-field. 


\section{E. Dual (disorder field) representation}

Whenever a field theory sustains topological defects, it is often useful to formulate a new field-theory of the topologial excitations of the original theory per se, and this forms a dual description of the original theory. We will do this for the present problem also, following Ref. 25. This means that the vortex-content Eq. [11] of the Ginzburg-Landau theory Eq. 5 in the phase-only Villain-approximation is cast into the form of a local field theory involving a complex scalar mass-field describing local vortex-fluctuations, coupled to a dual gaugefield that mediates an interaction between the vortexsegments. The resulting theory will exhibit explicitly a $U(1)$-symmetry, and as always in such cases, the question to be asked is under what circumstances, if any, the symmetry will be spontaneously broken 57 .

The purpose of this reformulation is to provide a point of contact between on the one hand a quantity to be introduced in Section IIIA and studied in Sections IVB and $\mathrm{VB}$, probing vortex-tangle connectivity and denoted $O_{L}$, and on the other hand thermodynamics. The key point is that in zero magnetic field, the two-point correlation function of the complex scalar mass-field $\phi(\mathbf{r})$ of the dual theory, is precisely the probability of finding a connected vortex path between the two points of the correlation function, regardless of by which path the two points are connected25. Long-range order in $G(\mathbf{x}, \mathbf{y})$ implies a broken $U(1)$-symmetry. Equivalently, long-range vortex connectivity in zero magnetic field implies a broken $U(1)$-symmetry, which is "hidden" at the level of Eq. 11, but is brought out when Eq. 11] is reformulated to the dual form, to be described below.

In three dimensions, and three dimensions only, a vortex-loop system interacting with a long-range BiotSavart interaction and steric repulsion, may in the continuum limit be written as a gauge thegry of a local complex matter field $\phi$ coupled to h25,58,18. We may extend the results of this work including fluctuations in $\mathbf{A}$ in a finite magnetic field, in which case the vortex-content of the Villain-approximation to the GL-theory corresponds precisely to an action of the following form

$$
\begin{aligned}
Z & =\prod_{\mathbf{r}} \int D \phi(\mathbf{r}) D \phi^{*}(\mathbf{r}) D \mathbf{h}(\mathbf{r}) D \mathbf{A}(\mathbf{r}) e^{\tilde{S}_{\mathrm{eff}}\left[\phi, \phi^{*}, \mathbf{h}, \mathbf{A}\right]} \\
\tilde{S}_{\mathrm{eff}} & =-\sum_{\mathbf{r}}\left[\alpha^{\prime}|\phi|^{2}+\frac{g^{\prime}}{2}|\phi|^{4}+\frac{1}{2}\left|\left(\frac{\nabla}{i}-e^{\prime} \mathbf{h}\right) \phi\right|^{2}\right. \\
& \left.+\frac{1}{2 \beta}(\nabla \times \mathbf{h})^{2}+2 i e(\nabla \times \mathbf{h}) \cdot \mathbf{A}+\frac{1}{2}(\nabla \times \mathbf{A})^{2}\right],
\end{aligned}
$$

where the coefficients $\left(\alpha^{\prime}, e^{\prime}, g^{\prime}\right)$ appearing in the theory are given in terms of the parameters entering Eq. 1158 . For our discussion, their precise values are of no importance. The interpretation of the $\phi$-field is that it is a local field describing local fluctuations in the topological excitations of the GL-theory, namely line-defects in the form of vortex-lines. An analogue of this dual description of the present $U(1)$-symmetric theory is the dual description of the Ising-model, where a local field is introduced to describe the local fluctuations in the topological defects of that model, which are domain walls separating different spin-ordered regions of the magnet. The effective action, Eq. 12, is invariant under the set of transformations

$$
\begin{aligned}
& \phi \rightarrow \phi \exp \left(i \omega_{\mathrm{h}}\right) \\
& \mathbf{h} \rightarrow \mathbf{h}+\frac{1}{e^{\prime}} \nabla \omega_{\mathrm{h}} \\
& \mathbf{A} \rightarrow \mathbf{A}+\nabla \omega_{\mathrm{A}} .
\end{aligned}
$$

By rewriting the theory in Eq. 11 in this way one observes that it explicitly exhibits a $U(1)$-symmetry. Note that this relies entirely on the possibility of reformulating the interacting loop-gas, including Biot-Savart interactions, in terms of a complex matter field $\phi$ coupled to a gauge-field $\mathbf{h}$.

Consider now for the moment the case of zero magnetic field. The main advantage of the above formulation is that the probability of finding a connected path of vortex segments, starting at $\mathbf{x}$ and ending at $\mathbf{y}, G(\mathbf{x}, \mathbf{y})$, is given by the two-point correlation function of the $\phi$-field 25

$$
G(\mathbf{x}, \mathbf{y})=<\phi^{*}(\mathbf{x}) \phi(\mathbf{y})>.
$$

A vortex-loop unbinding will lead to a finite $G(\mathbf{x}, \mathbf{y})$ when $|\mathbf{x}-\mathbf{y}| \rightarrow \infty$, because infinitely large loops will connect opposite sides of the vortex system. On the other hand, if $\lim _{|\mathbf{x}-\mathbf{y}| \rightarrow \infty} G(\mathbf{x}, \mathbf{y}) \neq 0$, this implies that $\left\langle\phi^{*}(x)>\neq 0\right.$, corresponding to a broken $U(1)$-symmetry. Therefore, the dual field $\phi(\mathbf{r})$ is an order parameter of a vortex-loop unbinding transition. The broken $U(1)$-symmetry is associated with the loss of number conservation of connected vortex-paths threading the system in any direction (including direction perpendicular to an applied magnetic field, if that is present). This limit of the two-point correlation function is closely related to the quantity $O_{L}$ we introduce in Section IIIA, which probes the connectivity of the vortex tangle in an extreme type-II superconductor. The above connection makes it at the very least plausible that an abrupt change in this connectivity, as probed by the change in $O_{L}$, is associated with breaking a $U(1)$-symmetry of the vortex-sector of the GL-theory, equivalently an onset $\left\langle\phi^{*}\right\rangle$ or $\langle\phi\rangle$. Since this only happens above a critical temperature, we may view $\phi$ as a disorder-field, in contrast to the order-parameter field $\psi$ of the original GL-theory. We will make explicit use of this connection in Section IV.

In zero magnetic field, we will show that the loss of superfluid density, specific heat anomaly, change in vortexloop distribution, loss of long-wavelength vortex-line tension, and abrupt change in vortex tangle connectivity abruptly occurs precisely at the same temperature both for the $3 D X Y$-model, also when amplitude fluctuations are included.

At finite magnetic fields, the situation is complicated by the fact that the vortex system is always connected 
across the system in at least one direction, namely the field direction, at all temperatures. One may however still extract information of the type encoded in $<\phi^{*}(\mathbf{r}) \phi(\mathbf{r})>$ at zero field by performing a singular gauge-transformation of the type used in Ref. 15, 18 , which roughly speaking amounts to subtracting out the field-induced vortices and studying the remaining loopgas, which has a field-theory description very much like the zero-field version of Eq. 12. The obvious advantage of this is that one removes the asymmetry of the system imposed by the magnetic field. A twopoint correlator of this theory then probes the connectivity of non-field induced vortex paths across the system, which in turns probes the possibility of having a broken $U(1)$-symmetry and hence an onset of the order parameter $\langle\phi(\mathbf{r})\rangle \neq 0$.

We will perform a numerical analogue of this in our simulations, namely we will probe the connectivity of the vortex tangle of the superconductor in directions perpendicular to the magnetic field. Ideally, what one should do is to generate phase-configurations (and vortex-configurations) of the extreme type-II superconductor, subtract out from each configuration a number of vortex paths that connects the system along the field direction precisely corresponding to the number of field induced vortices in the system, which is a fixed number in a canonical ensemble usually studied for this problem. Out of the remaining vortex tangle one may then try to find vortex paths connecting opposite sides of the system. Numerically this procedure is entirely prohibitive and we therefore opt for the algorithm of calculating $O_{L}$, to be described in detail in Section IIIA.

We stress that the procedure of computing $O_{L}$ described in Section IIIA unquestionably probes the connectivity of a vortex-tangle across the system, not associated with magnetic field, precisely as in the zero-field case. The objective is to probe the breaking of a $U(1)$ symmetry associated with the proliferation of unbound vortex-loops in the system, as pointed out in Ref. 43 . This will be shown to be precisely borne out in zero magnetic field. In finite magnetic field we also obtain a weak specific heat anomaly at the temperature where $O_{L}$ changes abruptly, as the system size is increased.

\section{DEFINITIONS, SIMULATION PROCEDURE, AND MODEL PARAMETERS}

In this section, we define the physical quantities considered, describe our Monte Carlo procedure, and present the values of the model parameters used in the simulations.

\section{Specific heat $C$}

To calculate the specific heat per site $C$, we use the fluctuation formula,

$$
\frac{C}{k_{B}}=\frac{1}{\mathcal{V}} \frac{<H^{2}>-<H>^{2}}{\left(k_{B} T\right)^{2}} .
$$

Here, the dimensionless volume $\mathcal{V}=L_{x} L_{y} L_{z}$, and $L_{\mu}$ is the dimensionless linear dimension of the system along the $\mu$-direction. $L_{\mu}$ is measured in units of the lattice constant $a_{\mu}$. As a check of consistency, we also calculate the specific heat per sitersing the numerical derivation of the internal energy $U 59,13,60$,

$$
C_{U}=\frac{1}{\mathcal{V}} \frac{\partial U}{\partial T}
$$

Note that for the FG model, where the effective Hamiltonian depends explicitly on the temperature, there strictly speaking is an additional term in the expression for the internal energy 13 ,

$$
U=-\frac{\partial \ln Z}{\partial \beta}=<H>-T\left\langle\frac{\partial H}{\partial T}\right\rangle .
$$

For the 3DXY model, if the simulations are properly done, $C \cong C_{U}$. For the FG model, where the effective Hamiltonian explicitly depends on the temperature, $C \neq C_{U}$. Note however that $<\partial H / \partial T>$ arises out of introducing a temperature dependence of the coefficients of the GL-theory 6159.13 . The temperature dependence of these coefficients always has a temperature dependence set by the mean-field critical temperature. Thus, close to the true $T_{c}$ these corrections, arising from integrating out the fermions of the underlying microscopic description of the superconductor 61 , are always negligible compared to the contribution coming from the true critical fluctuations of the order parameter, i.e. the transverse phasefluctuations. In terms of Eq. 8 the term $-T<\partial H / \partial T>$ orginates from the temperature dependence of the amplitude of the order-parameter. Were this to actually vanish at $T=T_{c}$, substantial corrections to the specific heat and entropy would result. As we will see later, at $T=T_{c}$, the ensemble average of the amplitude of the order parameter is far from renormalized to zero by vortex-loop fluctuations. Hence, at the critical point the correction term $-T<\partial H / \partial T>$ in the internal energy, with its resulting corrections to entropy 59 , is negligible 13 . There is now ample experimental evidence that critical fluctuations are indeed important over a sizeable temperature window in the $T_{c}$ cuprates of order several Kelvin below $T_{c} 38,39.62,63,40$ a window which is consistent with a coherence length of order $10 \AA$, about two orders of magnitude shorter than in conventional superconductors.

\section{Local Cooper-pair density $<\left|\psi^{\prime}\right|^{2}>$}

A. Definitions

As a probe for the local Cooper-pair density, we calculate 


$$
<\left|\psi^{\prime}\right|^{2}>\equiv \frac{1}{\mathcal{V}} \sum_{\mathbf{r}}<\left|\psi^{\prime}(\mathbf{r})\right|^{2}>
$$

We see in Eq. 17 that $\left\langle\left|\psi^{\prime}\right|^{2}\right\rangle$ involves both thermal and space average. Recall that $\psi^{\prime} \equiv \psi / \sqrt{|\alpha(0)| / g}$. At the mean field level, we expect $\left\langle\left|\psi^{\prime}\right|^{2}>\right.$ to develop an expectation value below the mean field critical temperature $T_{M F}(B)$.

\section{Superfluid condensate density $\left|\left\langle\psi^{\prime}\right\rangle\right|^{2}$}

As a probe for the local condensate density (density of Cooper pairs participating in the superconducting condensate), we calculate

$$
\left.\left.\left|<\psi^{\prime}\right\rangle\right|^{2} \equiv \frac{1}{\mathcal{V}} \sum_{\mathbf{r}}\left|<\psi^{\prime}(\mathbf{r})\right\rangle\right|^{2} .
$$

Note the difference between $<\left|\psi^{\prime}\right|^{2}>$ and $\left|\left\langle\psi^{\prime}\right\rangle\right|^{2}$. The former describes local Cooper-pair density, while the latter describes what is commonly known as the condensate density in ${ }^{4} \mathrm{He}$-physics. In zero field, we expect $\left.\left|<\psi^{\prime}\right\rangle\right|^{2}$ to develop an expectation value below the critical temperature $T_{c}$.

\section{Distribution of the order field phase angle}

To probe the distribution of the phase angle in $\psi^{\prime}(\mathbf{r})=$ $\left|\psi^{\prime}(\mathbf{r})\right| e^{i \theta(\mathbf{r})}$, we define the distribution function

$$
D_{\theta}\left(\theta^{\prime}\right)=\frac{1}{\mathcal{V}}<\sum_{\mathbf{r}} \delta_{\theta(\mathbf{r}), \theta^{\prime}}>
$$

Here, $\delta_{i, j}$ is the Kronecker delta function. In the simulations, we have chosen to work with a discrete set of phase angles, $\theta^{\prime}, \theta(\mathbf{r})=2 \pi n / N_{\theta}$. Here, $n \in\left[0, N_{\theta}\right]$ is an integer, and $N_{\theta}$ is the number of allowed discrete phase angles. In our experience, the simulation results do not depend on $N_{\theta}$, provided $N_{\theta} \gtrsim 16$. In zero field, when the phase is disordered, we expect $D_{\theta}(\theta)$ to be uniformly distributed, $D(\theta)=1 / N_{\theta}$. In the ordered phase, we expect $D_{\theta}(\theta)$ to show a peak around a preferred phase angle.

\section{Helicity modulus $\Upsilon_{\mu}$}

To probe the global superconducting phase coherence across the system, we consider the helicity modulus $\Upsilon_{\mu}$, defined as the second derivate of the free energy with respect to an infinitesimal phase twist in the $\mu$ direction 64,65.17. Finite $\Upsilon_{\mu}$ means that the system can carry a supercurrent along the $\mu$-direction. Within the 3DXY-model, the helicity modulus along the $\mu$-direction becomes,

$$
\begin{aligned}
\frac{\Upsilon_{\mu}}{J_{\mu}} & =\frac{1}{\mathcal{V}}\left\langle\sum_{\mathbf{r}} \cos \left[\Delta_{\mu} \theta-\mathcal{A}_{\mu}\right]\right\rangle \\
& -\frac{J_{\mu}}{k_{B} T \mathcal{V}}\left\langle\left[\sum_{\mathbf{r}} \sin \left[\Delta_{\mu} \theta-\mathcal{A}_{\mu}\right]\right]^{2}\right\rangle .
\end{aligned}
$$

For the FG case,

$$
\begin{aligned}
\frac{\Upsilon_{\mu}}{J_{\mu}}= & \frac{1}{\mathcal{V}}\left\langle\sum_{\mathbf{r}}\left|\psi^{\prime}(\mathbf{r})\right|\left|\psi^{\prime}(\mathbf{r}+\hat{\mu})\right| \cos \left[\Delta_{\mu} \theta-\mathcal{A}_{\mu}\right]\right\rangle \\
- & \frac{J_{\mu}}{k_{B} T \mathcal{V}} \times \\
& \left\langle\left[\sum_{\mathbf{r}}\left|\psi^{\prime}(\mathbf{r})\right|\left|\psi^{\prime}(\mathbf{r}+\hat{\mu})\right| \sin \left[\Delta_{\mu} \theta-\mathcal{A}_{\mu}\right]\right]^{2}\right\rangle .
\end{aligned}
$$

Note the difference between $\left.\left|<\psi^{\prime}\right\rangle\right|^{2}$ and $\Upsilon_{\mu}$, they are not identical. The former quantity probes the superfluid condensate density, which is a locally defined quantity, while the latter quantity probes a global phase coherence along a given direction $\mu$. Since $\left\langle\psi^{\prime}\right\rangle$ is the order parameter of the Ginzburg-Landau theory, close to the critical point we have

$$
\left|<\psi^{\prime}>\right|^{2} \sim|\tau|^{2 \beta}
$$

where $\tau=\left(T-T_{c}\right) / T_{c}$. On the other hand, $\Upsilon_{\mu} \propto \rho_{s \mu}$, where $\rho_{s \mu}$ is the superfluid density in the $\mu$-direction. Using the Josephson scaling relation $\rho_{s \mu} \sim \xi^{2-d} \sim$ $|\tau|^{\nu(d-2)}$ along with the scaling laws $\gamma=\nu(2-\eta)$ 6 and $2 \beta=2-\alpha-\sqrt{68}$, we find

$$
\Upsilon_{\mu} \sim|\tau|^{2 \beta-\eta \nu}
$$

Here, $d$ is the dimensionality of the system, $\nu$ is the correlation length exponent of the system, $\beta$ is the order parameter exponent, $\gamma$ is the order parameter susceptibility exponent, and $\eta$ is the anomalous dimension of the order parameter two-point correlation function at the critical point. Therefore, although $\left|<\psi^{\prime}>\right|^{2}$ and $\Upsilon_{\mu}$ are in principle different, they may appear to be very close if the anomalous dimension $\eta$ of the $\psi$-field is small, as indeed is the case for the Ginzburg-Landau theory, where $\eta \approx 0.0469$. Note that for $\eta>0$, the curve for $\Upsilon_{\mu}$ should bend more sharply towards zero at the critical point than $\left|<\psi^{\prime}>\right|^{2}$. We will explicitly show by direct calculations within the Ginzburg-Landau theory that $\left|\left\langle\psi^{\prime}\right\rangle\right|^{2}$ is very close to $\Upsilon_{\mu}$ both in zero field and finite magnetic field. In zero magnetic field this is precisely what one would expect based on the above, when $\eta<<170$. For the special case of $d=3$, we have $2 \beta-\eta \nu=\nu<2 \beta$. To high precision, we haye for the $3 D X Y$-model, that $\nu=0.673$ and $\eta=0.03869$ 


\section{Vortex loop distribution $D(p)$}

To probe the typical perimeter $L_{0}(T)$ and the effective long-wavelength vortex-line tension $\varepsilon(T)$ (not to be confused with the flux-line tension, which is always zero when gauge-fluctuations are completely suppressed due to the absence of tubes of confined magnetic flux), we define a vortex-loop distribution function $D(p)$, which measures the ensemble-averaged number of vortex loop in the system having a perimeter $p[1,17,14,36$. In order to compare results from different system sizes, we normalize $D(p)$ with respect to the system size.

We search for a vortex loop using the following procedure. Given a vortex configuration, we start with a randomly chosen unit cell with vortex segments penetrating its plaquettes. We follow the directed vortex path and record the trace. When the directed vortex path encounters a unit cell containing more than one outgoing direction, we choose the outgoing direction randomly. When the vortex path encounters a previously visited unit cell, i.e. when it crosses its own trace, we have a closed vortex loop, its perimeter being $p$. We now delete the vortex loop from the vortex configuration, to prevent double counting, and continue the search. The search is continued until all vortex segments are deleted from the system.

Using a 3D non-interacting boson analogy to the vortex system, it can be shown 72 that the distribution-function $D(p)$ can be fitted to the form 73

$$
D(p)=A p^{-\alpha} \exp \left[-\frac{\varepsilon(T) p}{k_{B} T}\right]
$$

Here, $\mathrm{A}$ is a temperature independent consfant, and the exponent $\alpha \approx 5 / 2$ to a first approximation 74 . When $\varepsilon(T)$ is finite, there exists a typical length scale $L_{0}=k_{B} T / \varepsilon$ for the thermally excited vortex loops. The probability of finding vortex loops with much larger perimeter than $L_{0}$ is exponentially suppressed, according to Eq. 22. When $\varepsilon=0, D(p)$ decays algebraically, and the length scale of the problem $L_{0}=k_{B} T / \varepsilon(T)$, has diverged. As a consequence, configurational entropy associated with topological phase-fluctuations is gained without penalty in free energy. In zero field, there is only one critical point, and in this case $L_{0}$ must be some power of the superconducting coherence length $\xi(T)$.

\section{Probe of vortex-connectivity, $O_{L}$}

For probing the connectivity of a vortex tangle in a type-II superconductor, in zero as well as finite magnetic field, we introduce a quantity $O_{L}$, defined in zero magnetic field as the probability of finding a vortex configuration that can have at least one connected vortex path threading the entire system in any direction. In the presence of a finite magnetic field, $O_{L}$ is defined as the probability of finding a similarly connected vortex path in a direction transverse to the field direction, without using the PBC along the field direction. In zero field, we use the same procedure as in finite-field, namely searching for connected vortex paths perpendicular to the $z$-direction, although in this case we could just as well have used any direction. Note that $O_{L}$ is very different from the winding number $W$ in the 2D boson analogy 75,47. There, $W$ is proportional to the number of vortex paths percolating the system transverse to the field direction. However, in the calculation of $W$, the $\mathrm{PBC}$ along the field direction is used many times.

In an attempt to probe "vortex-percolation", a slightly different quantity than $O_{L}$ has been considered in the context of high-temperature superconductors by others 76 . A7. A crucial difference between our work and that of Ref. 76, is that Ref. 76 allows periodic boundary conditions along the field direction to be used several times before the vortex path winds once around the $x$ or $y$-axis, as is easily seen from Fig. 2b of Ref. 76. This ultimately is the same as computing the winding number of the $2 D$ non-relativistic boson-analogy of the vortexsystemb, as recently done in careful Monte-Carlo simulations in Ref. 60. It also explains why the authors of Ref. 76 get longitudinal dissipation at the onset of what they denote "vortex-percolation", which is nothing but the temperature at which the winding number becomes finite.

This is entirely consistent with a number of other menterlo simulation results on the $3 D X Y$ mode 12, 17, 13, 43, 14 which all show the loss of longitudinal phase-coherence and onset of longitudinal dissipation precisely at the vortex lattice melting transition. This is measured simply by the helicity modulus $\Upsilon_{z}$, which is quite different from $O_{L}$. To the contrary, in our calculation of $O_{L}$, we do not allow for the use of periodic boundary conditions in the $z$-direction to measure vortex-tangle connectivity in the $x$ - or $y$-directions, in other words the "percolating" configurations of Fig. 2b of Ref. 76 are not counted when computing $O_{L}$.

We have

$$
O_{L}=\frac{N_{c}}{N_{\text {total }}}
$$

$N_{\text {total }}$ is the total number of independent vortex configurations provided by the Monte Carlo simulation. Furthermore, $N_{c}$ is the number of vortex configurations containing at least one directed vortex path that traverses the entire system perpendicular to the direction, without using the $\mathrm{PBC}$ along the field direction. For convenience, we treat the zero field case as the $\operatorname{limit} \lim _{B \rightarrow 0}$ keeping the "field direction" intact.

We search for the possibility of finding a vortex path such as described above by using the following procedure. Assume that the magnetic induction points along the $z$ axis. We follow all paths of directed vortex segments starting from all four boundary surfaces with surface normal perpendicular to $\hat{z}$, and check whether at least one 
of these vortex paths percolates the system and reaches the opposite surface, without applying the PBC in the $z$ direction. Note that when crossing vortex segments are encountered, the procedure is to attempt to continue in a direction that will bring the path closer to the opposite side of the system, rather than randomly resolving the intersection. $O_{L}$ is therefore a necessary, but not sufficient condition for finding an actual vortex-path crossing the system. However, in zero field this procedure does not make a difference to that of resolving the intersections randomly. This is demonstrated by the correlations of the change in $O_{L}$ and $D(p)$, to be detailed in the next section.

If a vortex path is actually found crossing the system in any direction in zero field, or without using $\mathrm{PBC}$ in the field-direction when a field is present, one may safely conclude that the vortex-line tension has vanished. If it were finite, it would not be possible to find such a path at all, either because all vortex-lines form closed confined loops in zero field, or because the vortex-line fluctuations along the field direction would be diffusive in finite field. In zero-field, this is clear by the above mentioned correlation between the change in $O_{L}$ and $D(p)$, cf. the results of the next section. In this paper, we also investigate this in detail for the finite-field case, by considering the position of the lowest temperature $T_{L}$ where we have $O_{L}=1$ both as a function of system size and aspect ratio $L_{x} / L_{z}=L_{y} / L_{z}$. If vortex-line physics remains intact, $T_{L}$ should move monotonically up with system size, and should scale with $L_{x} / L_{z}$. Instead, we will find that $T_{L}$ moves down slightly, and saturates with increasing system size at fixed aspect ratio. In addition, we find that $T_{L}$ is virtually independent of aspect ratio for large enough systems.

This contradicts expectations based on a lines-only approximation to the vortex-liquid. It demonstrates that the connectivity of the vortex-tangle undergoes a fundamental change inside the vortex-liquid. The above mentioned finite size scaling analysis, suggests to us that this geometric transition is a property that survives in the thermodynamic limit. The issue is whether the change in connectivity has anything to do with a thermodynamic phase-transition. This will be investigated in detail for zero magnetic field in Section IIIA, and for finite magnetic field in Section IIIB. In particular, we look for a specific heat anomaly scaling up with system size, at the putative transition point $T_{L}$. This will reveal if the change in the geometric properties of the vortex liquid is indeed associated with singular thermodynamics. In any case, once the geometric transition has taken place, it is no longer possible to model the vortex-liquid regime in terms of field-induced flux lines only, with merely renormalized interactions between them.

In the VLL phase $O_{L}=0$, since the field induced flux lines are well defined and do not "touch" each others, and the thermally excited vortex loops are confined to sizes smaller than the magnetic length 17 . $O_{L}=1$ in the normal phase above the crossover region where the rem- nant of the zero field vortex loop blowout takes place. Needless to say, it is a matter of interest to investigate precisely where $O_{L}$ changes value from zero to one.

Note that $O_{L}$ itself is not a genuine thermodynamic order parameter, although it may be said to probe an order-disorder transition 4 . However, by the transcription of the vortex-content of the Ginzburg-Landau theory to the form Eq. 12 in Section IIF, it is brought out that probing the vortex-tangle connectivity by considering $O_{L}$ is closely connected to probing the two-point correlator of a local complex field $\phi(\mathbf{r})$, the dual field of the local vorticity-field $m_{\mu}(\mathbf{r})$ of the Villain-approximation and London-approximation to the Ginzburg-Landau theory, Eqs. 11. The two-point correlator $\left\langle\phi^{*}(\mathbf{r}) \phi\left(\mathbf{r}^{\prime}\right)\right\rangle$ is ultimately the probe of whether or not the $\phi$-theory Eq. 12 exhibits off-diagonal long-range order and a broken $U(1)$-symmetry. An entirely equivalent interpretation of the change in $O_{L}$ was given in Ref. 43 which did not involve a local field $\phi(\mathbf{r})$, but number conservation of vortex-lines threading the entire superconductor. This number is conjugate to the phase-field of the local complex field $\phi(\mathbf{r})$. An advantage of the present formulation involving Eq. 12 is that it directly relates he change in $O_{L}$ to the long-distance part of a correlation for a local field, and hence to a local order parameter $\langle\phi(\mathbf{r})\rangle$. This connection makes it at least plausible that the change in vortex-tangle connectivity, i.e. a change in the geometry of the vortex-tangle, may be related to a thermodynamic phase-transition. We emphasize that the present problem is very different from the percolation transition known to occur in the $3 D$ Ising-model, and which has nothing to do with the thermodynamic phase-transition in that mode 8 .

\section{Structure function $S(\mathbf{k})$}

To probe the structure of the VLL, we consider the structure functipn for vortex segments directed along the field direction 9 . 16. Given an applied field along $z$-axis, the structure function $S(\mathbf{k})$ is defined by

$$
S(\mathbf{k})=\frac{<\left|\sum_{\mathbf{r}} n_{z}(\mathbf{r}) \exp [i \mathbf{k} \cdot \mathbf{r}]\right|^{2}>}{(f V)^{2}} .
$$

Here, $\mathbf{k}$ is a reciprocal lattice vector, and the filling fraction $f$ is defined in Eq. 26. In the VLL phase, $S\left(\mathbf{k}_{\perp}, k_{z}=0\right)$ has $\delta$-function Bragg peaks at $\mathbf{k}_{\perp}=\mathbf{K}$, where $\mathbf{K}$ is reciprocal lattice vector for the ordered VLL. When the VLL melts, the Bragg peaks are smeared out $S\left(\mathbf{K}, k_{z}=0\right)$ drops to a very small value. Thus, the VLL melting temperature can be defined as the temperature where $S\left(\mathbf{K}, k_{z}=0\right)$ shows a sharp drop to a value very close to zero. In a liquid where we have full rotational invariance, we expect $S\left(\mathbf{k}_{\perp}, k_{z}=0\right)$ to exhibit a ring pattern, with a very small amplitude. 


\section{Extended Landau gauge:}

Periodic boundary conditions together with Landau gauge

$$
\mathcal{A}_{y}=2 \pi f x
$$

give rise to a constraint, $L_{x} f=1,2,3 \ldots$ Thus, for given $L_{x}$, the smallest $f$ allowed is $f=1 / L_{x}$. To perform simulations and finite size scaling of systems with very low filling fractions, we define an "extended" Landau gauge,

$$
\mathcal{A}_{x}=\frac{2 \pi y m_{y} m n}{L_{x} L_{y}} ; \mathcal{A}_{y}=\frac{2 \pi x n_{x} m n}{L_{x} L_{y}}
$$

where $n_{x}, n, m_{y}, m$ are positive integers satisfying $n_{x} n=$ $L_{y}$, and $m_{y} m=L_{x}$. The filling fraction $f$ is now given by

$$
f=\frac{n m\left[n_{x}-m_{y}\right]}{L_{x} L_{y}} .
$$

Hence, it is possible to chopse systems with a filling fraction as low as $f=1 / L_{x} L_{y} 43$.

\section{B. Details of the Monte-Carlo simulations}

The statistical mechanics of the 3DXY model and the FG model is investigated by Monte-Carlo simulations on the effective Hamiltonians Eq. 10 and Eq. 7.

For the 3DXY model, a Monte-Carlo move is an attempt to replace a phase angle at a given site $\theta(\mathbf{r})$ with a new randomly chosen phase angle $\theta^{\prime} \in[0,2 \pi>$. For the FG model, a Monte-Carlo move is an attempt to replace a complex number at a given site $\psi(\mathbf{r})$ with a new randomly chosen complex number $\psi^{\prime}$. Here, $\left|\psi^{\prime}\right| \in[0: 1+\epsilon]$ and $\theta^{\prime} \in[0,2 \pi>$. We have introduced a small positive parameter $\epsilon$ to allow the system to perform Gaussian fluctuations, around the extremal field configuration $\left|\psi^{\prime}\right|^{2}=1$, at very low temperature. Note that we are letting the amplitude fluctuate around its mean value at every temperature. The Monte-Carlo move is accepted or rejected according to the standard Metropolis algorithm 80 . If the new phase angle causes a gauge invariant phase differences $j_{\mu}=\Delta_{\mu} \theta-\mathcal{A}_{\mu}$ to fall outside the primary interval $[-\pi, \pi>$, we take it back into the primary interval. This compactization procedure creates a closed unit vortex loop around the link where $j_{\mu}$ is changed. In this way, all the vortex loops introduced into the system are closed, and the net induction is always conserved.

A Monte-Carlo sweep consists of $L_{x} \times L_{y} \times L_{z}$ MonteCarlo moves. Typical runs consist of $1.2 \times 10^{5}$ sweeps per temperature, where the first $2 \times 10^{4}$ sweeps are discarded for equilibration. Near the phase transitions up to $2 \times 10^{6}$ sweeps per temperature is necessary to capture the correct physics. For a given system, we always start the simulation by a cooling sequence, where the starting temperature is significantly higher than all temperatures associated with phase-transitions or crossovers the model might exhibit. The results shown in this paper originate both from cooling and heating sequences. Since these two methods give essential identical results, we do not differentiate between them.

In order to resolve anomalies in the specific heat, we must in some cases perform simulations on systems as large as $360^{3}$. To be able to carry out simulation on such large systems, we must 1) write part of the code in assembly and 2) carry out the simulations in a parallel manner. Our systems are divided into "black and white" subsystems, arranged in a 3D checkerboard pattern. Each black subsystem has only six white subsystems as its nearest neighbors, and visa versa. Since the $3 \mathrm{DXY}$ and the FG model only have nearest neighbor interactions, all subsystems with the same color can be updated simultaneously.

To be able to calculate a nonparallel-able routine as $O_{L}$ in an effective manner, we divide the computer nodes in 2 groups, the large main group takes care of the Monte Carlo simulation, and a small subgroup carries out, simultaneously, the calculation of $O_{L}$.

\section{Model parameters}

a. System sizes: We put our coordinate $(\mathrm{x}, \mathrm{y}, \mathrm{z})$-axes along the crystal (a,b,c)-axes. For the anisotropic cases, we assume uniaxial anisotropy, and use the crystal $c$ axis as the anisotropy axis. We perform simulations on tetragonal systems with dimensions $L_{x}, L_{y}, L_{z}$. The main part of the simulations is done on cubic or nearly cubic systems. Nearly cubic systems $L_{x} \sim L_{y}=L_{z}$ is some times necessary in order to satisfy the boundary conditions enforce by the extended Landau gauge, Eq. 25. To check for the finite size effect of $O_{L}$, we carry out simulations on slab systems with the aspect ratios $L_{y} / L_{z}=1.00,1.25,1.5,1.75,2.00$. System sizes up to $360^{3}$ were used.

b. Cooper-pair chemical potential $\alpha(T)$ : . We let the Cooper-pair chemical potential have the simple linear form

$$
\frac{\alpha(T)}{\alpha(0)}=\frac{T-T_{M F}}{T_{M F}} .
$$

We have also carried out simulations with other forms for $\alpha(T) / \alpha(0)$, such as $\tanh \left(\left[T-T_{M F} / T_{M F}\right] T_{0}\right)$. Here, $T_{0}$ is a constant regulating the size of the region where $\alpha(T) / \alpha(0)$ grows from -1 to 1 . The results are, however, qualitatively the same as for the linear case. The parameter $T_{M F}$ is the parameter effectively controlling the width of the critical region in these calculations. In units of $J_{0}$, Eq. 8, we write $T_{M F}^{\prime}=k_{B} T_{M F} / J_{0}$. The values we will use are $T_{M F}^{\prime}=0.3,1.0$. An estimate for what temperatures these values correpond to may readily be obtained by using $a_{z}=11 \AA, \lambda_{a b}=1500 \AA$, implying that $T_{M F}^{\prime}=1$ corresponds to $300 K$, while using $\lambda_{a b}=2000 \AA$ implies that $T_{M F}^{\prime}=1$ corresponds to 
$180 K$. These are very reasonable numbers. With these parameters, it will be shown that amplitude fluctuations of the local Ginzburg-Landau order parameter, when included on an equal footing with the phase-fluctuations, are far from being critical.

c. Anisotropy parameter $\Gamma$ : The anisotropy parameter $\Gamma$ is defined as

$$
\Gamma=\frac{a_{z} \xi_{x}}{a_{x} \xi_{z}}=\frac{\lambda_{c} a_{z}}{\lambda_{a b} a_{x}} .
$$

Note that $\Gamma>1$ only when the layering of the superconductor to be simulated is pronounced, i.e. $d_{\mu}>\xi_{\mu}$ for at least one direction $\mu$. In this article, we consider systems with the anisotropy parameter $\Gamma=1,3,7$.

d. Filling fraction $f$ : The filling fraction along the $\mu$-direction, $f_{\mu}$, is defined as

$$
2 \pi f_{\mu}=(\Delta \times \mathcal{A})_{\mu}
$$

$f_{\mu}$ is a measure of the fraction of flux quanta of magnetic induction penetrating a single plaquette with surface normal along $\hat{\mu}$. When the magnetic field is applied along $z$-axis, $f_{x}=f_{y}=0$, and

$$
f \equiv f_{z}=\frac{B a_{x} a_{y}}{\Phi_{0}} \quad \stackrel{3 D X Y}{=} \quad \frac{B \xi_{a b}^{2}}{\Phi_{0}} .
$$

In this work, we consider filling fractions $f=$ $0,1 / 20, \ldots . ., 1 / 1560$.

\section{MONTE-CARLO SIMULATIONS, B =0}

In this subsection, we discuss the zero field superconducting-normal (S-N) phase transition, both in terms of the usual Ginzburg-Landau order field $\psi(\mathbf{r})$, and in terms of the behavior of topological excitations which can be tied to the formulation of the transition using the disorder-field picture presented in Section IIF. We compare our results obtained from the FG mgdel to known simulation results of the $3 \mathrm{DXY}$ model 113 , the London model 31, and the Villain model 82.17.

Unless otherwise stated, in this subsection we show simulation results for the FG model with the parameters: $f=0, \Gamma=1, T_{M F}^{\prime}=0.3,1, a_{\mu} / \xi_{\mu}=6$, and $\mathcal{V}=60^{3}$. We have chosen $a_{\mu} / \xi_{\mu}=6$ to slightly enhance the critical features of the FG model. Simulations of the FG model using a smaller ratio $a_{\mu} / \xi_{\mu}=4$ leads to the same conclusions, but larger systems and longer simulation times are required to obtain the same quality of the data. What we will find is that the width of the regions where phase-fluctuations dominate is controlled by the parameter $T_{M F}$, increasing with $T_{M F}$. Our picture of the zero-field transition as a vortex-loop unbinding is however borne out regardless of the value of $T_{M F}$, close enough to the true critical temperature $T_{c}$.

\section{A. Order field}

First we present results for the S-N phase transition in terms of an order field picture, i.e. in terms of the ordinary local pair-wave function $\psi(\mathbf{r})$ of the GinzburgLandau theory. We obtain results of the full GL theory including amplitude and phase-fluctuations. What will be shown, even when amplitude fluctuations are included, is that we obtain a clear picture of the S-N phase transition in terms of an Onsager-Feynman vortex loop unbinding 8385 , driven exclusively by topological phasefluctuations of the Ginzburg-Landau order parameter.

In Fig. 11 we plot the helicity modulus $Y_{z}$, the local density of Copper pairs $<\left|\psi^{\prime}\right|^{2}>$, and the superfluid condensate density $\left|\left\langle\psi^{\prime}\right\rangle\right|^{2}$ as functions of temperature. We see that the condensate density $\left|\left\langle\psi^{\prime}\right\rangle\right|^{2}$ is zero above a critical temperature and develops a finite expectation value below $T_{c}$. In contrast to this, $\left\langle\left|\psi^{\prime}\right|^{2}\right\rangle$ is finite both above and below $T_{c}$. Close to $T=T_{c}$ we have performed the simulations for a very dense set of temperatures, and from the top panel of Fig. 1, we may however discern a kink in the curve and hence a singular behavior of the temperature derivative of $\left\langle\left|\psi^{\prime}\right|^{2}>\right.$. The top panel shows the results for $T_{M F}=1.0$, while the lower panel shows the same results for $T_{M F}=0.3$. The difference between the two panels is that since $T_{M F}$ has been changed, the width of the critical region has changed, increasing upon increasing $T_{M F}$. Had we chosen $T_{M F}=0.01$, an appropriate value for conventional superconductors, the curves for $\left.\left|<\psi^{\prime}\right\rangle\right|^{2}$ and $<\left|\psi^{\prime}\right|^{2}>$ would have been indistinguishable, the conventional $B C S$ mean-field picture of the superconducting transition would have been appropriate. The reason that it is no longer the case in the high- $T_{c}$ cuprates is the large energy scale for pairing, coupled with the fact that the phase-stiffness is low.

Note how the curve for $\Upsilon_{z} \sim|\tau|^{2 \beta-\eta \nu}$ bends slightly more sharply towards zero than the curve for the condensate density $\left|\left\langle\psi^{\prime}\right\rangle\right|^{2} \sim|\tau|^{2 \beta}$, as expected for a positive $\eta$, since in that case $2 \beta-\eta \nu<2 \beta$. In fact, this provides a nice consistency-check on the Monte-Carlo simulations.

We may therefore conclude that the existence of Cooper pairs does not imply superconductivity, and the local Cooper pair density can not be used as a probe of the superconducting phase. Rather, the superconducting phase is characterized by a finite expectation value of the superfluid condensate density, i.e $\left\langle\psi^{\prime}(\mathbf{r})\right\rangle \neq 0$. Recall that $\left.\left|<\psi^{\prime}\right\rangle\right|^{2} \neq 0$ only if $\left\langle\psi^{\prime}(\mathbf{r})\right\rangle \neq 0$. The slow decay of $\left\langle\left|\psi^{\prime}\right|^{2}>\right.$ above the true superconducting transition temperature $T_{c}$ is due to gaussian fluctuations in the amplitude of the order parameter.

The results shown in Fig. 11 justify, quite clearly, that the neglect of amplitude fluctuations in order to study the critical behavior, is an entirely appropriate approach to this problem. In the normal phase, the phase angle of the order field is uniformly distributed, Fig. 1 inset, while for $T<T_{c}$, the system spontaneously chooses a preferred phase angle giving a peak in $D_{\theta}(\theta)$. Due to 
our finite set of discrete phase angles, $D_{\theta}(\theta)=1 / N_{\theta}$ for $T>T_{c}$, and not zero as in the continuum- $\theta$ limit.
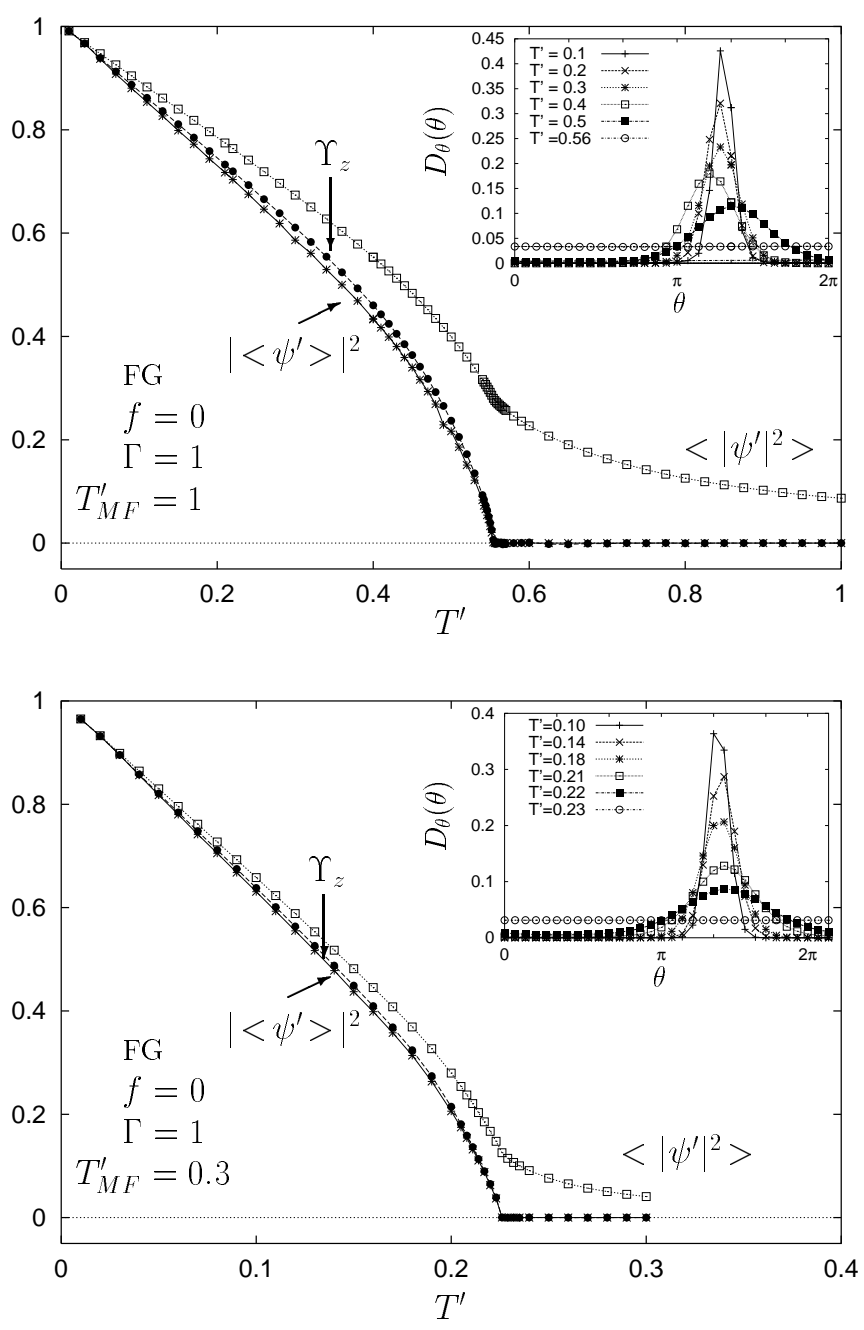

FIG. 1. Helicity modulus $\Upsilon_{z}$, local Cooper-pair density $<\left|\psi^{\prime}\right|^{2}>$, and superfluid condensate density $\left.\left|<\psi^{\prime}\right\rangle\right|^{2}$ as functions of temperature for the Ginzburg-Landau model in a frozen gauge approximation. Upper panel shows results for $f=0, \Gamma=1, T_{M F}^{\prime}=1.0, a_{\mu} / \xi_{\mu}=6$, and $\mathcal{V}=60^{3}$. Lines are guide to the eye. $\Upsilon_{z}$ and $\left|\left\langle\psi^{\prime}\right\rangle\right|^{2}$ develop finite expectation values for $T<T_{c}$, while $\left\langle\left|\psi^{\prime}\right|^{2}\right\rangle$ is finite both above and below $T_{c}$. Inset: The distribution function of the phase angle of the order field $D_{\theta}(\theta)$ as a function of $\theta$, for several temperatures. Below $T_{c}$, a preferred phase angle is chosen and the global $U(1)$ symmetry is spontaneously broken. Note how the phase fluctuates around a mean value even in the ordered phase. Lower panel shows the same for $T_{M F}^{\prime}=0.3$. The width of the critical region has decreased, but the amplitude fluctuations are still far from being critical.

Note the difference between this picture compared to the usual mean field picture of the S-N phase transition. In the mean field picture, the phase angle of the order field does not fluctuate. Thus, $\left\langle\left|\psi^{\prime}\right|^{2}\right\rangle=\left|\left\langle\psi^{\prime}\right\rangle\right|^{2}$, and $T_{c}$ can be defined as the highest temperature where the local Cooper pair density $\left\langle\left|\psi^{\prime}\right|^{2}>\right.$ develops a finite expectation value. This happens, at the mean field level neglecting all fluctuations in $\psi^{\prime}$, at $T_{M F}$ where the Ginzburg-Landau parameter $\alpha(T)=1-T^{\prime} / T_{M F}^{\prime}$ becomes negative. Including fluctuations $<\left|\psi^{\prime}\right|^{2}>\neq \mid<$ $\psi^{\prime}>\left.\right|^{2}$, and $T_{c}$ is defined as the lowest temperature where the superfluid condensate density $\left|\left\langle\psi^{\prime}\right\rangle\right|^{2}$ still maintains a value of zero.

Given that the condensate density is non-zero below $T_{c}$, we next focus on a global quantity, the longwavelength limit of the helicity modulus $\Upsilon_{\mu}$, or equivalently the superfluid stiffness in the $\mu$-direction. In Fig. 1 we see that $\Upsilon_{z}$ vanishes for temperatures $T \geq T_{c}$, and develops an expectation value for $T<T_{c}$. Thus, the superconducting phase exhibits global phase coherence, while the normal phase does not. We have also calculated $\Upsilon_{x}$ and $\Upsilon_{y}$, and found (not shown) that they show the same behavior as $\Upsilon_{z}$. Apart from minor details, we see in Fig. I that the helicity modulus is proportional to the condensate density 64. We will also show that this equality also applies to the finite field case.

At low temperature, $\Upsilon_{\mu}$ decreases linearly. This feature is also obtained in the zero field $3 \mathrm{DXY}$ model13, but not in the zero field Villain model-17. In the Villain model, the spin waves and the vortex loops can be analytically decoupled. Here, at low temperatures, spin wave excitations do not affect the vortex loops excitations and the superfluid phase stiffness should decay in an activated manner due to the excitation of vortex loops. In the 3DXY model, the spin wave and the vortex loops are coupled together. Whether or not the low-temperature features of $\Upsilon_{\mu}$ in Fig. I can explain experimental data on the temperature dependence of $1 / \lambda_{\mu}^{2}$, see for instance Ref. 36, is an interesting but o far unsettled issue, see also the results of Refs $13,43,22$. Within the anisotropic $3 D X Y$-model, the helicity modulus $\Upsilon_{z}$ has a larger, but negative slope of its linear low- $T$ behavior compared to $\Upsilon_{x}$ and $\Upsilon_{y}$. On the other hand, it is not entirely trivial to connect $\Upsilon_{z}(T) / \Upsilon_{z}(0)$ to the $T=0$-normalized superfluid density $\rho_{s z} 8$, which is the quantity measured in the experiments of Hardy et al.86. However, our main point of emphasis is that the vanishing of the superconducting phase stiffness at $T=T_{c}$ is caused exclusively by an unbinding of large vortex loops. Further evidence for the connection between $\Upsilon_{\mu}$ and the vortex loops can be found in simulations of the lattice London model where vortexloops are the only degrees of freedom $31,19.88$. Here, the normalized helicity modulus $\lim _{k \rightarrow 0} \Upsilon_{\mu}(T) / \Upsilon_{\mu}(T=0)$ is renormalized to zero at $T_{c}$ exclusively by the expansion of vortex loops.

The critical behavior of the $3 D X Y$-model including coupling between spin-waves and vortices, is the same as for the vortex-content of the same theory, but taken in the Villain-approximation, where no coupling between vortices and spin-waves exist. The only excitations in the $U(1)$-symmetric gauge-theories in the frozen gauge- and amlpitude-approximation, are longitudinal and trans- 
verse phase-fluctuations, i.e. spin-waves and vortexloops.

The above considerations and results provide an overwhelming amount of evidence in favor of the proposition that unbound vortex-loops are precisely the critical fluctuations of an extreme type-II superconductor.

Approaching $T_{c}$ from below, $\Upsilon_{\mu}(T)$ decays to zero with an exponent consistent with $v=2 \beta-\eta \nu$, and $\eta \approx 0.04$ as discussed in Section III A.5, see Fig. 11. For the special case of $d=3$, which we consider, we have $\rho_{s \mu} \sim \xi^{-1} \sim|\tau|^{\nu}$, and hence we find $2 \beta-\eta \nu=\nu<2 \beta$. We will show in the next section that this renormalization of $\Upsilon_{\mu}$ is nicely explained by the expansion and blowout of thermally excited vortex loops.

\section{B. Topological excitations and vortex-line tension}

We now discuss the $\mathrm{S}-\mathrm{N}$ phase transition in terms of the behavior of topological objects of the FG model, i.e. closed vortex loops and vortex lines.

The first figure of this section shows our probe of the vortex-tangle connectivity, $O_{L}$, as a function of temperature, for various system sizes and $\Gamma=1$ and 7 . We reemphasize that $O_{L}$ is not suggested as an order parameter of the transition, but that it nevertheless can be tied to a local order parameter via the formulation of the theory given in Section IIF, unlike the known spin-percolation transition in the $3 D$ Ising-model 88 .

In Fig. 2, we show the probability of finding a connected vortex-tangle across the system in zero magnetic field, for $\Gamma=1,7$, and system sizes $L^{3}$, with $L=6, . .64$. Notice how the curves cross at approximately the same temperature and get progressively sharper. Similar results were seen for considerably smaller system sizes $L=4,6,8$ in Ref. 89. Below, we will also give results for much larger system sizes, confirming that the crossing temperature in Fig. 2 gives a good estimate for the threshold temperature for vortex-loop unbinding throughout the sample. As pointed out in Ref. 89, such a finite-size effect indicates that a percolation threshold exists for the vortex tangle in the thermodynamic limit 90 . A precisely similar finite-size effect in $O_{L}$ will be seen in finite magnetic field, to be considered in Section VB. This will happen inside the vortex liquid phase at elevated magnetic fields, but will coincide with VLL melting at low fields, and suggests the revision of the picture of the molten phase of the Abrikosov vortex system purely in terms of a vortex-line liquid.

We next proceed to correlate the change in $O_{L}$ with the unbinding of large-vortex loops and the loss of vortex-line tension at $T=T_{c}$, by correlating its abrupt change with the characteristics developing in $D(p)$, which probes the typical size of thermally induced vortex loops in the system. We first consider the case of the $3 D X Y$-model, for which the results are shown in Fig. 3. The top panel shows specific heat, $O_{L}$ and helicity modulus, while the bottom panel shows the vortex-loop distribution function $D(p)$ as a function of perimeter $p$ for a number of temperatures $T \leq T_{c}$, while the inset of the bottom panel shows the temperature dependence of the longwavelength vortex-line tension $\varepsilon(T)$. In the top panel it is clear that the loss of helicity modulus, the anomaly in specific heat, and the abrupt change in $O_{L}$ all occur at precisely the same temperature. The change in the decay of $D(p)$ also occurs at the same temperature, $T_{c}$.
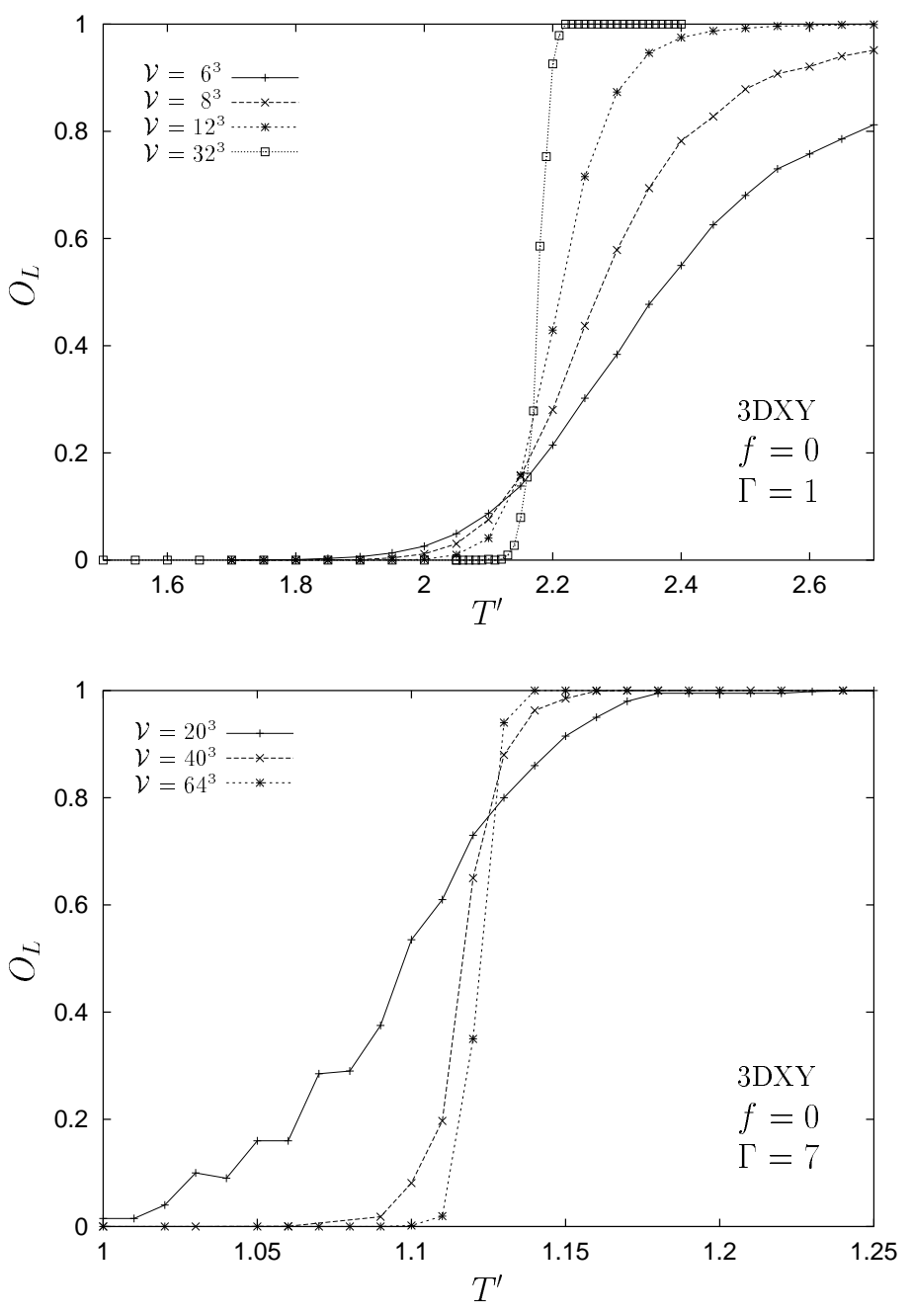

FIG. 2. $O_{L}$ as a function of temperature for several system sizes for the $3 \mathrm{DXY}$ model with $\mathrm{f}=0$. Lines are guide to the eye. Top panel: $\Gamma=1$, bottom panel: $\Gamma=7$. Note the finite-size effect in $O_{L}$, with the crossings of the curve approximately at the same temperature. 

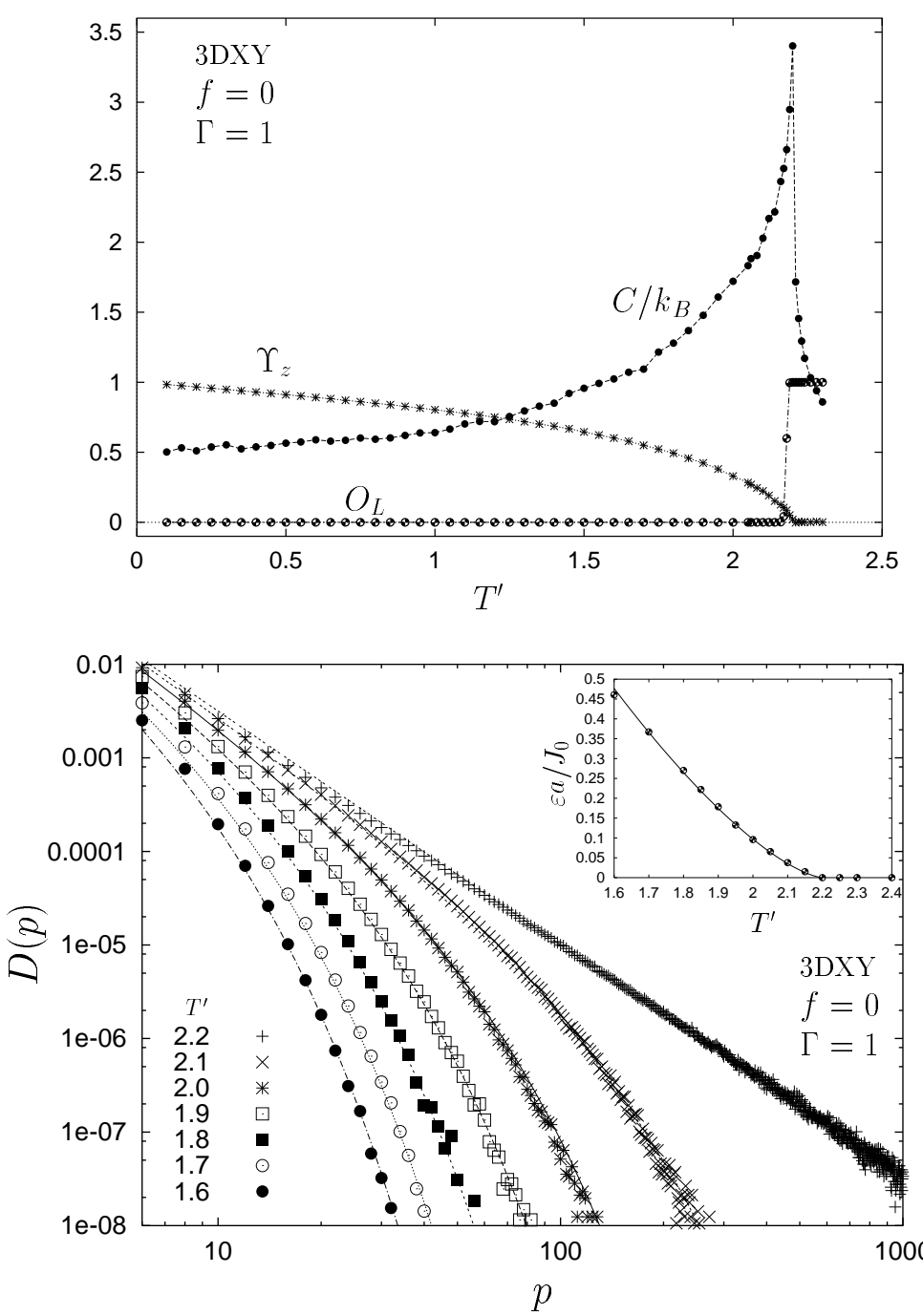

FIG. 3. Top panel: Specific heat $C$, helicity modulus $\Upsilon_{z}$, and $O_{L}$ as functions of temperature for the 3DXY model with $f=0, \Gamma=1$ and $\mathcal{V}=120^{3}$. Lines are guide to the eye.

Bottom panel: Vortex-loop distribution function $D(p)$ as a function of loop-perimeter $p$ for various temperatures. Lines are fits using $D(p)=p^{-5 / 2} \exp \left(-\varepsilon(T) p / k_{B} T\right)$. At $T=T_{c}$ the decay changes from exponential to algebraic implying that the vortex-line tension $\varepsilon$ vanishes. Inset of bottom panel shows $\varepsilon(T)$. Solid line is a fit using $\left|T^{\prime}-T_{c}^{\prime}\right|^{\gamma}$, with $\gamma=1.45 \pm 0.05$.

For $T<T_{c}, O_{L}=0$, and all vortex loops are confined, with typical size given by $\mathrm{E}_{0}(T)=k_{B} T / \varepsilon(T)$, where

$$
L_{0}(T)=\frac{k_{B} T}{\varepsilon(T)}
$$

where $\varepsilon(T)$ is the effective long-wavelength vortex line tension, equivalently the free energy per unit length of vortex-lines. These objects, present also in the lowtemperature phase, cause only a local perturbation of the order parameter in the system, and may simply be "coarse grained" away. The low energy physics of the model is therefore described essentially by the physics of the zero temperature fixed point. At and above $T_{c}$, $O_{L}=1$, and vortex loops with infinite size always exist. The length scale $L_{0}(T)$ has diverged, showing that there are vortex-loops on all length-scales with a power-law tail in the distribution. Such loops cannot be coarse grained away and taken into account by any "appropriate renormalization" of the zero-temperature theory. Thus, the $S-N$ phase transition can be viewed as a blowout out of thermally induced vortex loops. Above $T_{c}$, free thermally induced "vortex lines" exist in all direction, and any infinitesimal applied current will move these thermally induced "vortex lines" and dissipate energy 91 . Thus, the system is in the normal phase.
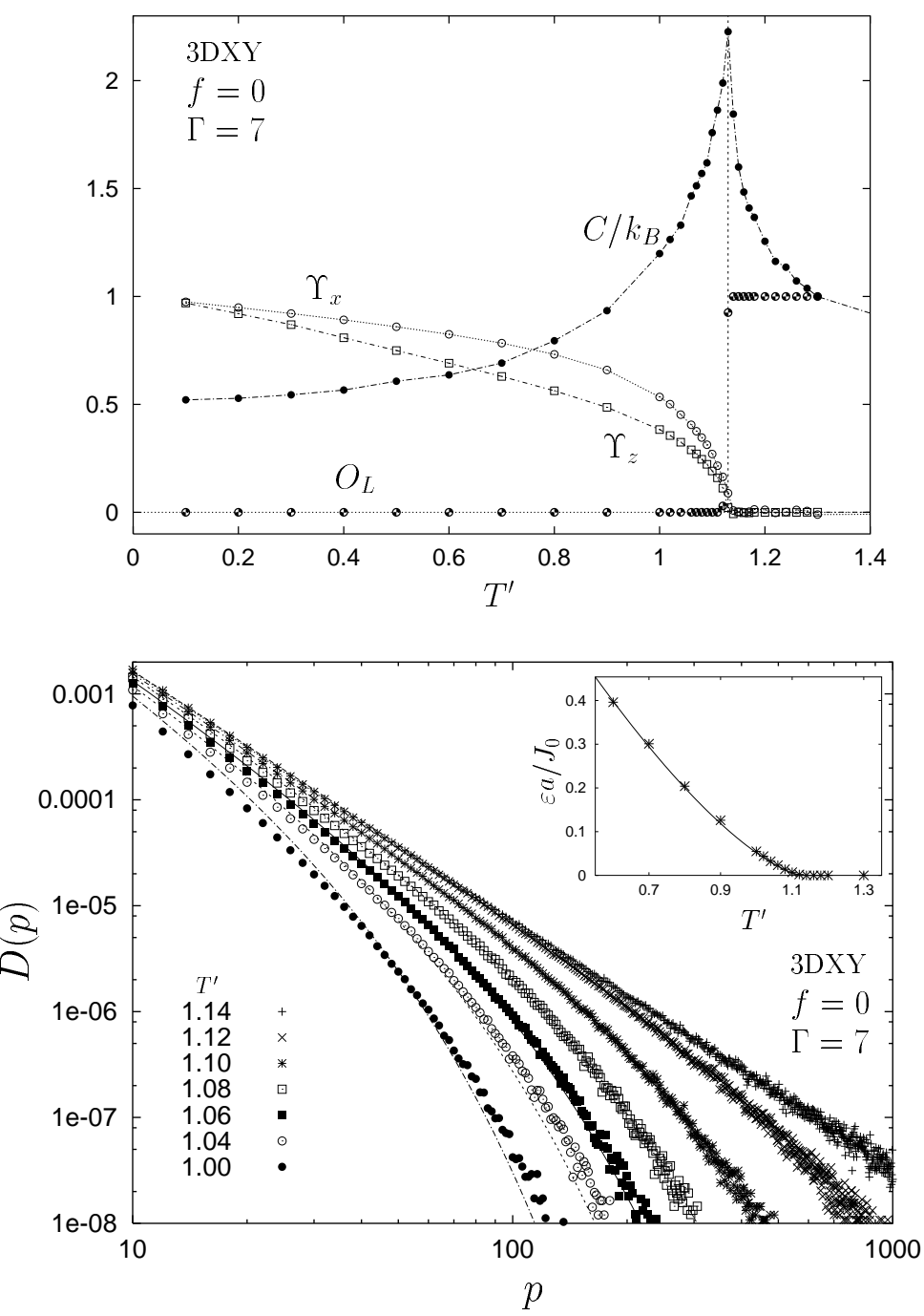

FIG. 14. Top panel: Specific heat $C$, helicity moduli $\Upsilon_{x}, \Upsilon_{z}$, and $O_{L}$ as functions of temperature for the $3 \mathrm{DXY}$ model with $f=0, \Gamma=7$, and $\mathcal{V}=140^{3}$. Lines are guide to the eye.

Bottom panel: Vortex-loop distribution function $D(p)$ as a function of loop-perimeter $p$ at various temperatures. Lines are fits using $D(p)=0.37 p^{-2.35} \exp \left(-\varepsilon(T) p / k_{B} T\right)$. Inset: Vortex-line tension $\varepsilon(T)$ as a function of temperature. Solid line is a fit using $\left|T^{\prime}-T_{c}^{\prime}\right|^{\gamma}$, with $\gamma=1.45 \pm 0.05$. 

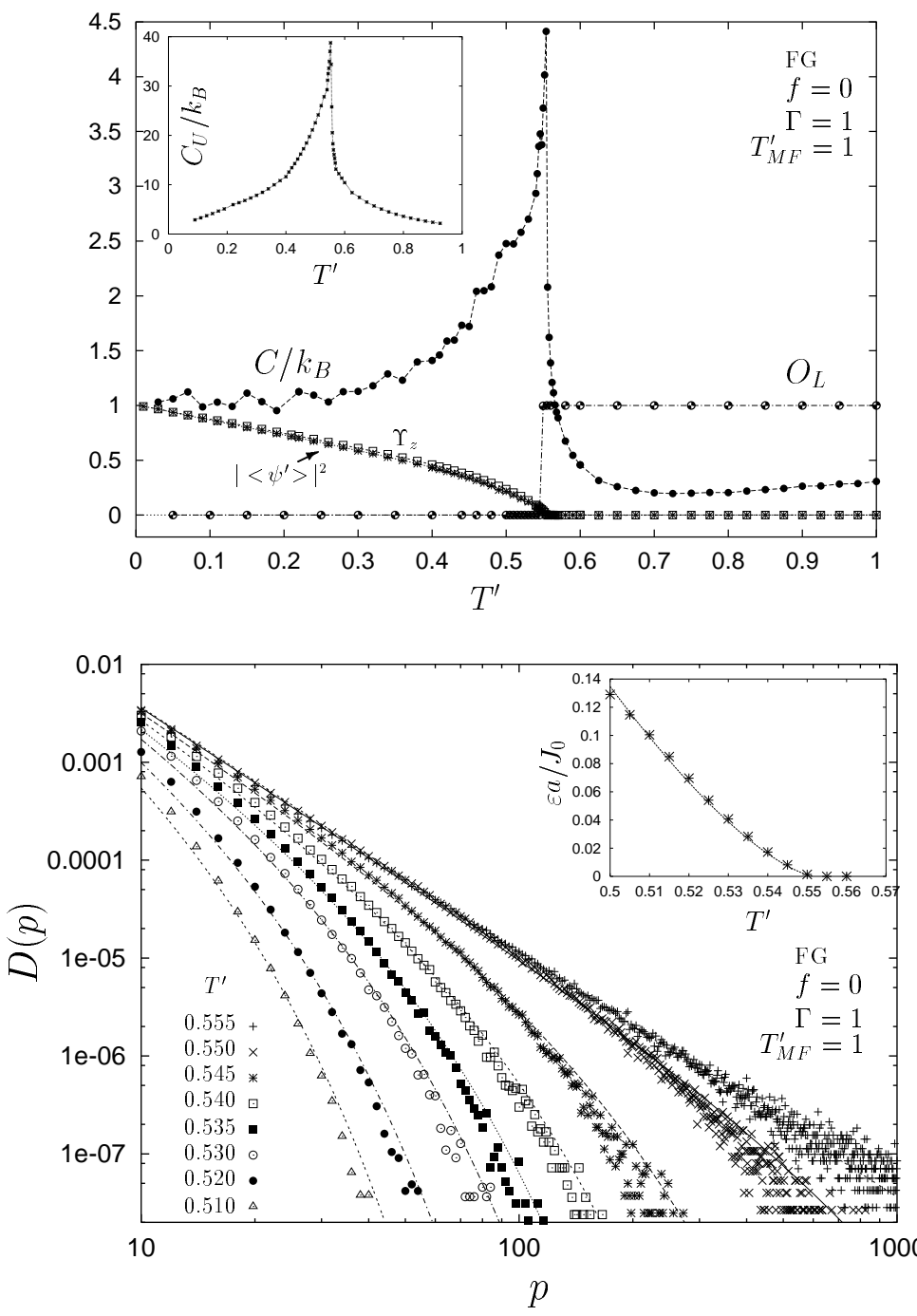

FIG. 5. Top panel: Specific heat $C$ calculated using Eq. 15, $O_{L}$, helicity modulus $\Upsilon_{z}$, and superfluid density $\left|<\psi^{\prime}>\right|^{2}$ as functions of temperature for the GinzburgLandau model in a frozen gauge approximation with $f=0$, $\Gamma=1, T_{M F}^{\prime}=1, a_{\mu} / \xi_{\mu}=6$, and size $\mathcal{V}=60^{3}$. Lines are guide to the eye. Inset shows the specific heat calculated using Eq. 16.

Bottom panel: Distribution of vortex loops $D(p)$ as a function of the loop-perimeter $p$ for several temperatures. Lines are fits using $D(p)=1.15 p^{-5 / 2} \exp \left(-\varepsilon(T) p / k_{B} T\right)$. Inset: Vortex-line tension $\varepsilon(T)$ as a function of temperature. Dotted line is a fit using $\left|T^{\prime}-T_{c}^{\prime}\right|^{\gamma}$, with $\gamma=1.45 \pm 0.05$.

In Fig. 4, we show the specific heat anomaly, the helicity moduli $\Upsilon_{x}$ and $\Upsilon_{z}$, as well as $O_{L}$ for the $3 D X Y$ model, with $\Gamma=7$. The correlation noted above in connection with Fig. 3 is again perfect, the only difference being that the specific heat anomaly has become more symmetric due to the increased anisotropy, $\Gamma=7$. Although the amplitude of $\Upsilon_{x}$ is larger than the amplitude of $\Upsilon_{z}$ due to the uniaxial anisotropy along the $z$-axis, the temperature at which they vanish, and the power law with which they vanish, are the same. Note also the sharpness of the manner in which the moduli $\Upsilon_{\mu}$ approach zero at $T_{c}$, there is no high-temperature tail as one would have found in too small systems. This in fact serves as a highly non-trivial benchmark on the quality of the Monte-Carlo simulations.
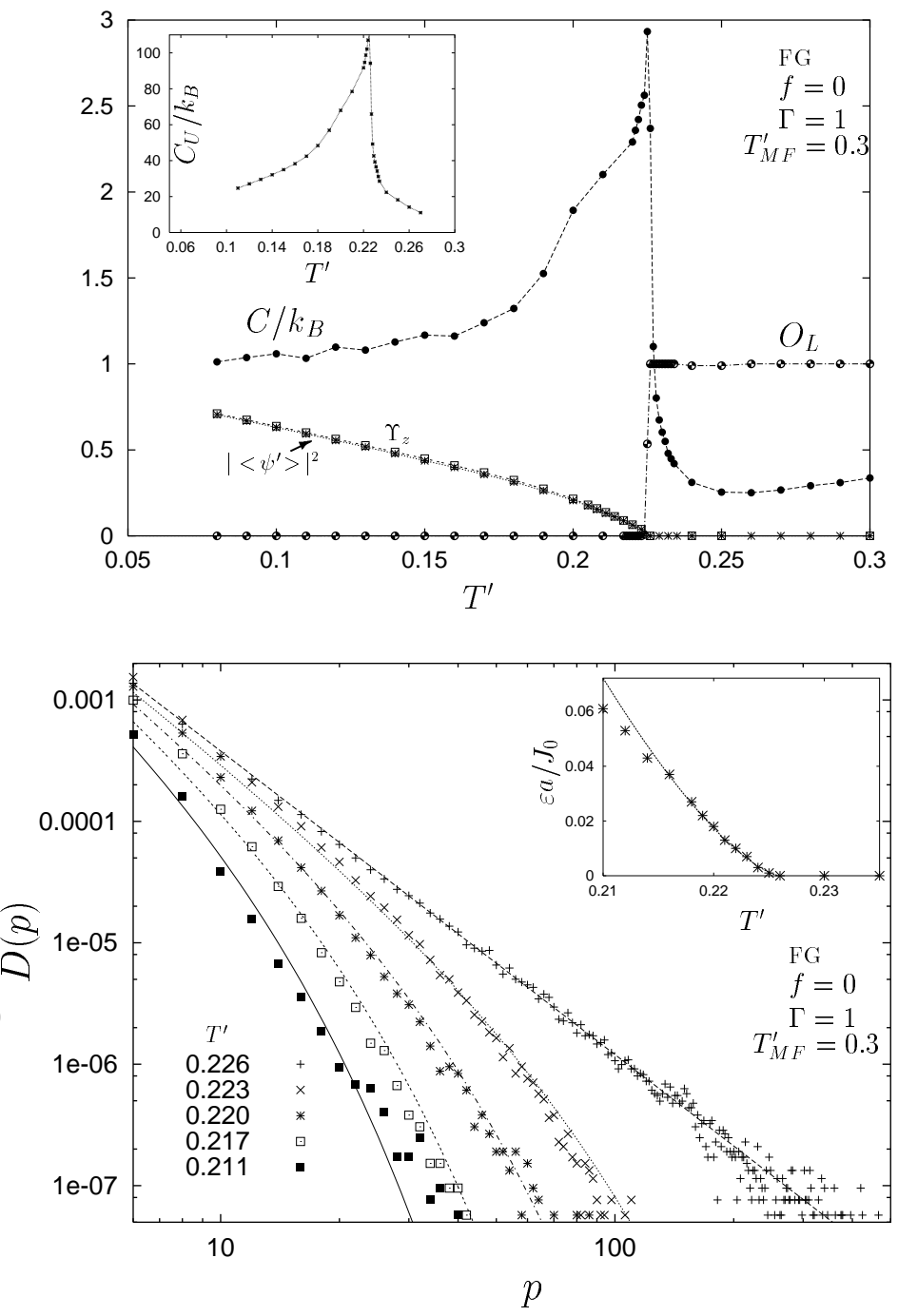

FIG. 6. Same as Fig. 目, with $T_{M F}^{\prime}=0.3, D(p)=$ $0.12 p^{-5 / 2} \exp \left(-\varepsilon(T) p / k_{B} T\right)$, and $\varepsilon(T) a / J_{0}=29\left|T^{\prime}-T_{c}^{\prime}\right|^{1.45}$.

Figs. 5 and 6 show essentially the same as Fig. 4 , but now for the FG model, i.e. including amplitude fluctuations on an equal footing with the phase-fluctuations. Clearly, the picture that it is the topological phasefluctuations, or the vortex-loop unbinding, that drives the superconductor normal-fluid transition, is not at all altered by the fact that amplitude fluctuations are included. This is a reconfirmation of the results obtained in Section IVA, showing that amplitude fluctuations of the local Ginzburg-Landau order parameter have a large mass at the critical temperature where the superfluid density vanishes. The vortex-loop unbinding, which is the microscopic mechanism driving the transition, cannot be 
reparametrized in terms of critical amplitude fluctuations of the local order parameter of the theory, since the latter are clearly nowhere close to being critical.

For a more detailed study of the properties of thermally induced vortex loops, we now focus on the vortex-loop distribution function $D(p)$ as a function of vortex-loop perimeter $p$ at various temperatures. These are shown in Figs. 3, 4, and 5 and are clearly well approximated by the form $D(p)=A p^{-5 / 2} e^{-\varepsilon(T) p / k_{B} T}$ for all temperatures considered. Note that $\varepsilon(T)$ is the only temperaturedependent fitting parameter in all plots. The effective long-wavelength linetension of vortex loops is finite below $T_{c}$, and vanishes for $T \geq T_{c}$. The physical picture of this phase transition is as follows. Below $T_{c}, \varepsilon(T)$ is finite defining a typical length scale for the vortex loops, $L_{0}=k_{B} T / \varepsilon(T)$. Here, $D(p)$ is dominated by an exponential decay and vortex loops with much larger perimeter $p$ than $L_{0}$, are exponentially suppressed. Thus, the topological excitations that are present in the system may be coarse grained away. At and above $T_{c}, \varepsilon(T)=0$ and no typical length scale for the vortex loops exist; the length scale $L_{0}$ has diverged. Here, $D(p)$ is purely algebraic, and vortex loops of all sizes including infinite size, exist. Thus, the S-N phase transition at $T_{c}$ is triggered by an unbinding of large vortex loops, analagous to the Onsager-Feynman mechanism, 17 mugested for the superfluid-normalfluid transition in ${ }^{4} \mathrm{He} \mathrm{B}^{4}$.

In the insets in the bottom panels of Figs. 3, 4, 5, and 6, we show the vortex line tension $\varepsilon(T)$ extracted from the vortex loop distribution function $D(p)$. Regardless of whether the $3 D X Y$ - or the FG-models are used, we find that the long-wavelength vortex-line tension vanishes as

$$
\varepsilon(T) \sim\left|T-T_{c}\right|^{\gamma} ; \quad \gamma=1.45 \pm 0.05 .
$$

The numerical value of the exponent $\gamma$ has been extracted from the systems with the largest critical regions, i.e. Figs. 3 , 田, and 5. The system shown in Fig. 6 does not allow a very precise value for $\gamma$ to be obtained, although the qualitative aspects of the results are clearly precisely the same as those for the $3 D X Y$-model and the FGapproximation of the GL-model with $T_{M F}^{\prime}=1.0$. This implies that the typical vortex-loop perimeter diverges when $T_{c}$ is approached from below, using Eq. 27, as

$$
L_{0}(T) \sim\left|T-T_{c}\right|^{-\gamma},
$$

such that $L_{0}(T)$ is a power of the correlation length $\xi$ of the $3 D X Y$ model.

\section{Anomalous dimension of the dual field}

We next connect the result for $\varepsilon(T)$ to the anomalous dimension of the dual field $\phi$. It is natural, within the formulation of the problem given in Section IIF, to associate the proliferation of unbound vortex-loops with a vortex-loop susceptibility, or equivalently a susceptibility for the $\phi$-field of Section IIF. This is seen as follows. The proliferation of unbounded vortex loops as the temperature of the superconductor is increased, is associated with the development of long-range correlations in the two-point correlation function of the dual field, $G(x) \equiv<\phi^{*}(x) \phi(0)>$, where on the low-temperature side the dual order parameter has zero expectation value, $<\phi>=0$. A scaling Ansatz for $G(x)$ reads

$$
G(x)=\frac{1}{|x|^{d-2+\eta_{\phi}}} \mathcal{G}(x / \xi),
$$

where $\eta_{\phi}$ is the anomalous dimension of the dual field $\phi, \xi$ is its correlation length, and $\mathcal{G}(x / \xi)$ is some scaling function. The square of the mass of the dual field, $m_{\phi}^{2}$, is therefore naturally mapped to the line-tension $\varepsilon(T)$ of the vortex-loops. This follows from the observation that the dual boson system of which the $\phi$-theory is a fieldtheory description, has a chemical potential $m_{\phi}^{2}$ which in turn is nothing but the line-tension $\varepsilon(T)$ of the vortexloop system, when the density distribution $D(p)$ is viewed as a partial density in a fugacity expansion for the density of the dual bose-system 72 . The Fourier-transform $\tilde{G}(k)=<\phi^{*}(k) \phi(-k)>$ of $G(x)$ may be written on form

$$
\tilde{G}(k)=\xi^{2-\eta_{\phi}} \quad \mathcal{F}(k \xi),
$$

where $\mathcal{F}(k \xi)$ is some new scaling function. The $k \rightarrow 0$ limit of this is the static uniform susceptibility $\chi_{\phi}$ of the dual field on the low-temperature side, where $\langle\phi\rangle=0$. On the other hand, as long as the dual field is massive, which it is on the low-temperature side, we must have $\lim _{k \rightarrow 0} \tilde{G}(k)=m_{\phi}^{-2}$. Hence, we obtain

$$
\chi_{\phi} \sim \frac{1}{m_{\phi}^{2}} \sim \frac{1}{\varepsilon} \sim \xi^{2-\eta_{\phi}} \sim|\tau|^{-\nu_{\phi}\left(2-\eta_{\phi}\right)} .
$$

The field $\phi$ has a correlation length exponent given by $\nu_{\phi}=2 / 3$, the same as for the $3 D X Y$-model 9 . This follows from the fact that it is a thermodynamic exponent describing the divergence of one and the same length in the Ginzburg-Landau theory and dual theory. Very importantly, it must be equal both for the dual model andits Ginzburg-Landau counterpart by "strong" duality 22 94. Were this not to hold, the dual of the dual theory would not be the original theory, as it ought to be. The above of course precisely amounts to the Fisher scaling-law 67 relating the susceptibility exponent of the dual field $\gamma_{\phi}$ to $\nu_{\phi}$ and $\eta_{\phi}$

$$
\gamma_{\phi}=\nu_{\phi}\left(2-\eta_{\phi}\right) .
$$

Using our estimate $\gamma_{\phi}=1.45 \pm 0.05$ with $\nu_{\phi}=2 / 3$ gives $\eta_{\phi}=-0.18 \mp 0.07$ in close agreement with previous renormalization group calculations 92 , who found $\eta_{\phi}=-0.20$ to one-loop order.

The result $\eta_{\phi}=-0.18 \mp 0.07$ obtained directly from computing the statistics of the loop-excitations of the $3 D X Y$-model is a truly noteworthy result, when viewed 
juxtaposed to the RG-calculations of Ref. 92. In Ref. 92, the RG-result for the anomalous dimension of the dual field was obtained directly from the dual theory. On the other hand, our numerical result is obtained directly from the phase-only approximation to the original GinzburgLandau theory. The agreement shows conclusively, and to our knowledge for the first time, that viewing the zerofield transition of the $3 D$ Ginzburg-Landau theory as a vortex-loop unbinding, which is the phase-transition of the dual theory, is precisely correct, not only qualitatively, but quantitatively 95 .

At and below $T_{c}$, the order field $\left\langle\psi^{\prime}(\mathbf{r})\right\rangle$ develops an expectation value, and explicitly breaks the global $U(1)$ symmetry of the GL-theory. In contrast to the order field picture, in a description using only topological excitations, the global $U(1)$ symmetry is hidden. There does not appear to be any symmetry operation involving the phase of a local field, that will leave the effective action Eq. 11 invariant. Therefore, there is also no obvious local quantity that develops an expectation value in the non-symmetric phase. Nevertheless, it is possible to define a global quantity that implicitly probes the breaking of the global $U(1)$ symmetry, namely $O_{L}$. Let $N_{\mu}$ denote the number of "vortex lines" (percolating directed vortex paths without using $\mathrm{PBC}$ ) along the $\mu$-direction. Below $T_{c}, N_{\mu}$ is fixed to zero and $O_{L}=0$. Concomitant with the conservation of the global quantity $N_{\mu}$, the system must exhibit a global $U(1)$ symmetry. At and above $T_{c}$, $N_{\mu}$ develops an expectation value and $O_{L} \neq 0$. This leads to a broken $U(1)$ symmetry.

\section{MONTE-CARLO SIMULATIONS, $\mathrm{B} \neq 0$}

We next discuss the indications we have of phasetransitions in the vortex-system in a finite magnetic field. In addition to the first order VLL melting transition line $T_{m}(B)$ which we map out for a large range of filling fractions, we find indications for a new phase transition in the vortex liquid. We emphasize that in all simulations performed in finite magnetic field, the filling fraction is low enough to ensure that there is zero transverse Meissner effect at any temperature of interest. That is to say, the vortex-line lattice is depinned from the numerical lattice at much lower temperature than the temperatures where the Bragg-peaks in the structure function of the VLL vanishes. Therefore, commensuration effects due to defining the theory on a lattice effectively have been eliminated at the temperatures of interest.

Before entering into the discussion, a clarifying remark is appropriate. Note that the phase-transition that we suggest may be taking place inside the vortex liquid, is not a transition from a disentangled low-temperature vortex liquid to an entangled high-temperature vortex liquid, as discussed by numerous previous authors. Such a transition would have has its hallmark that the superfluid stiffness along the magnetic field, or equivalently the he- licity modulus $\Upsilon_{z}$, would vanish inside the vortex-liquid phase. This has now been conclusively demonstrated not to be the case 1214.96.

At the phase transition we propose inside the vortex liquid, a global $U(1)$ symmetry is spontaneously broken. The probe for this new phase transition is, as for the zero field case, a sudden change in the connectivity of the vortex system, probed by $O_{L}$. As defined in the zero-field section, we have found that the vortex-loop distribution function $D(p)$ is not useful as a probe for the vortex line tension in finite field. Fortunately, non-local quantities with directions built-in as the helicity modulus, and the percolation probability still apply in finite field. To probe the VLL melting, we must define a new direction dependent non-local quantity, namely the structure function for vortex segments parallel to the field, $S(\mathbf{k})$. Quantities probing local properties as $\left\langle\left|\psi^{\prime}\right|>\right.$ and $\left|<\psi^{\prime}>\right|$ are still useful in finite field.

\section{A. First order VLL melting transition}

In this sub-subsection, we show and discuss our results with the focus on the first-order VLL melting transition.

We show in Fig. 7 the structure function for vortex segments along the field direction $S(\mathbf{K}=[\pi / 15,4 \pi / 15,0])$ and the fluctuation specific heat $C$ as functions of temperature for the FG model. Here, $\mathbf{K}$ is a reciprocal lattice vector of the VLL.

We see in Fig. 7 that $S(\mathbf{K})$ shows a sharp drop at $k_{B} T_{m} / J_{0}=0.26$ from $\sim 0.2$ to 0 , indicating a first order VLL melting transition. The Lindemann number for this melting transition estimated from the Debye-Waller factor at the melting temperature, is 0.25 , consistent with previous estimates $1,47.13$. This abrupt disappearance of the VLL structure fyption has also been experimentally found in BSCCO97,98. In the high-field regime, the position of the relting line is well estimated by the Lindemann-criterion 6.43 .

Density plots (not shown) of $S\left(\mathbf{k}_{\perp}, 0\right)$ show Bragg spots for $T$ below $T_{m}$, and rings pattern for $T$ just above $T_{m}$. Here, $\mathbf{k}_{\perp}=\left[k_{x}, k_{y}, 0\right]$ and $\left(k_{x}, k_{y}\right) \in[-\pi: \pi]$. This expected peak-to-ring feature is also found at $T_{m}$ in simulations on the 3DXY model 13 and in the Villain model65, 17. Below $T_{m}$, the vortex lines perform small Gaussian fluctuations around their equilibrium positions, and renormalize $S(\mathbf{K} ; T)$ to a smaller value compared to $S(\mathbf{K} ; T=0)$. Precisely at $T_{m}$ the specific heat shows a delta-function like anomaly, Fig. 7, indicating the wellestablished first-order character of the VLL-melting transition in clean systems 11 6 . Note that $T_{m} / T_{B c 2}$ increases for decreasing $T_{M F}$. For very small $T_{M F}$, where the mean field approximation applies, $T_{m} \simeq T_{B c 2}$. 

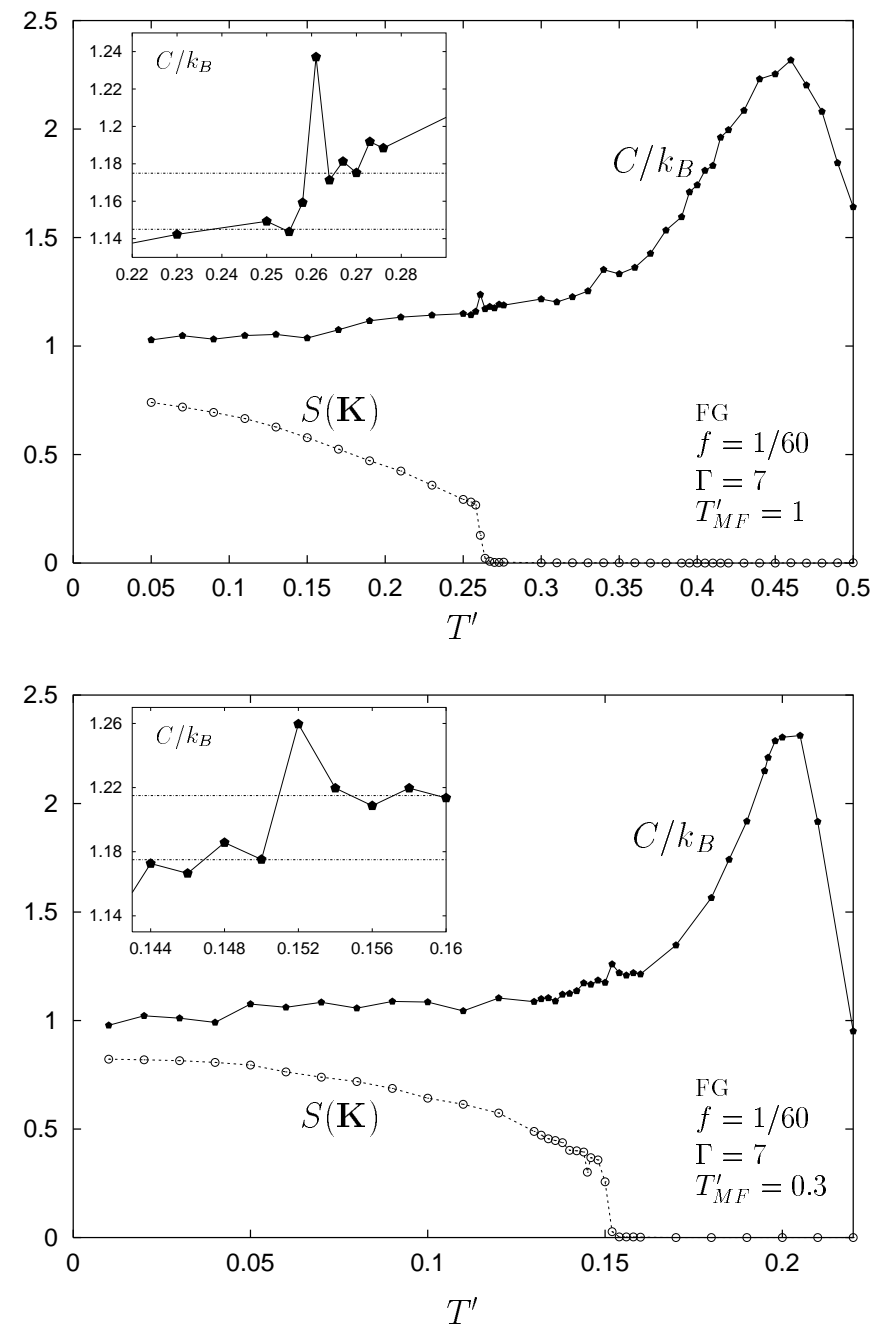

FIG. 7. Top panel: Structure function for vortex segments along the field direction $S(\mathbf{K}=[\pi / 15,4 \pi / 15,0])$ and specific heat $C$ as functions of temperature for the Ginzburg-Landau model in a frozen gauge approximation with $f=1 / 60, \Gamma=7$, $T_{M F}^{\prime}=1, a_{\mu} / \xi_{\mu}=6$, and $\mathcal{V}=60^{3} . S(\mathbf{K})$ shows a sharp drop at $k_{B} T_{m} / J_{0}=0.26$ indicating a first order VLL melting transition. $C$ shows a delta-function like anomaly at $T_{m}$, the VLL melting transition, detailed in the inset.

Bottom panel: Same as top panel, but for $T_{M F}^{\prime}=0.3$.

In Fig. 8, we show the local Cooper-pair density $<\left|\psi^{\prime}\right|^{2}>$, the local condensate density $\left|\left\langle\psi^{\prime}\right\rangle\right|^{2}$ and the helicity modulus along the field direction $\Upsilon_{z}$ as functions of temperature for the FG model.

We see in Fig. 8 that $\left\langle\left|\psi^{\prime}\right|^{2}\right\rangle$ does not show any particular anomalous feature at the melting temperature $T_{m}$, it is completely smooth. In contrast to this, $\left|\left\langle\psi^{\prime}\right\rangle\right|^{2}$ shows a sharp drop to zero at $T_{m}$, which indicates a first order phase transition at $T_{m} \cdot\left\langle\left|\psi^{\prime}\right|^{2}\right\rangle$ shows a small tail above $T_{m}$. However, note that this tail decreases towards zero for increasing simulation length. Thus, the superfluid condensate density is finite in the VLL phase, jumps discontinuously to zero at $T_{m}$, and is zero in the entire vortex liquid phase. The vortex-liquid phase is therefore phase-incoherent, and we denote the phase as an incoherent vortex liquid. The behavior of the helicity modulus shows that there is no signature of any transition from a disentangled to an entangled portex liquid phase, in agreement with previous results 12 . 13 . In the language of the non-relativistic $2 D$ boson-analogy commonly invoked in the study of vortex-liquids, there is no transition from a normal fluid to superfluid bose-system at $T=0$ of the bose-system, in agreement with general arguments given by Landau. In the absence of disorder, a normal bose-fluid cannot exist at $T=0$ in the thermodynamic limit, the only possible phases are bose-crystals or superfluids and hence there should be a direct transition from an insulating bose-system (corresponding to the Abrikosov vortex lattice phase) to a superfluid bosesystem, corresponding to an incoherent vortex liquid.

This is precisely what is observed in all our simulations, and demonstrates that all our systems are sufficiently large in the z-direction to capture this physics correctly. In Fig. 8, the helicity modulus along the field direction $\Upsilon_{z}$ also shows a sharp drop to zero at $T_{m}$. Thus, the vortex liquid is incoherent and can not carry any supercurrent. Note that $\Upsilon_{z}$ is a global quantity and says nothing about local (in space and time) phase coherence. Thus, even when $\Upsilon_{\mu}=0$, the system can exhibit local superconductivity, and diamagnetic response is expected in the incoherent vortex liquid.

It is interesting to note that while this comes out fairly straightforwardly in simulations on the $3 D X Y$ mode12, 13 , it is much more difficult to obtain using Quantum Monte Carlo simulations of the $2 D$ nonrelativistic boson-analogy of the lines-only approximation to the vortex-liquid 47.60 . This is due to the fact that the so-called winding number, which is the appropriate quantity to measure, is essentially inaccessible for large systems. One has to resort to computing quantities which are not the same as the winding number, but hoping that it is a representative of it. For a nice discussion of this point, see Refs. 47,60. It still has not been very well established precisely which fluctuations are responsible for destroying longitudinal phase-coherence at the VLL melting transition.

The loss of global phase coherence in the incoherent vortex liquid does not mean that the layers are decoupled. The correlation length $\xi_{\mu}(T)$, for all directions $\mu$, jumps from infinity to a finite value at $T_{m}$, and further decreases for increasing temperature. Estimates for various correlation lengths along the field direction can be found in Refs. 47,96. This picture nicely explains the experimental results found in BSCCO in Ref.99. Here, the difference in the high frequency resistivity, for different sample configurations, is used to probe the VLL melting transition. In the VLL phase $\xi_{\mu}$ is infinite in all directions, and the "rigid" VLL does not contribute to the dissipation when a high-frequency circulating current is applied (Fig. 2 in Ref.99) is applied. In the vortex liquid however, this dissipation depends on the correlation 
length in the different directions. Due to anisotropy and the applied field along the crystal's $c$-axis, $\xi_{c} \neq \xi_{a b}$, and the high-frequency microwave dissipation is different for different sample orientation. For increasing temperature, $\xi_{\mu}(T)$ decreases further and thus the dissipation rate increases as found in Ref. 99 .
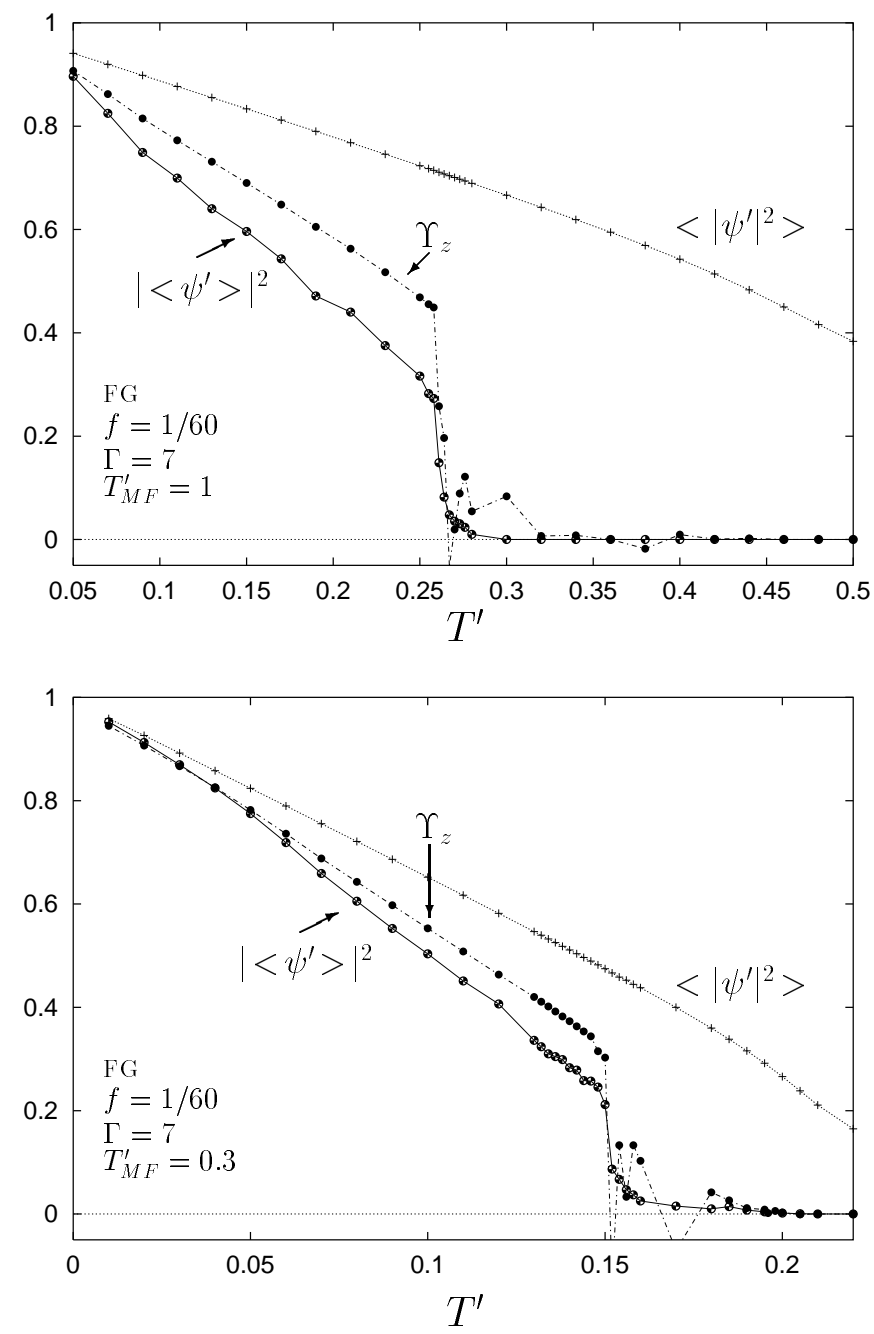

FIG. 8. Top panel: Local Cooper-pair density $\left\langle\left|\psi^{\prime}\right|^{2}\right\rangle$, local condensate density $\left|<\psi^{\prime}>\right|^{2}$ and helicity modulus along the field direction $\Upsilon_{z}$ as functions of temperature for the Ginzburg-Landau model in a frozen gauge approximation with $f=1 / 60, \Gamma=7, T_{M F}^{\prime}=1, a_{\mu} / \xi_{\mu}=6$, and $\mathcal{V}=60^{3}$. $\left|<\psi^{\prime}>\right|^{2}$ and $\Upsilon_{z}$ show a sharp drop at $T_{m}$, implying zero condensate density and lack of global phase coherence in the vortex liquid.

Bottom panel: Same as top panel, but with $T_{M F}^{\prime}=0.3$

In the past there have been simulations on the 3DXY model 89,100 , the Villain model65, and the London model 101. 16 that suggest a two stage "melting transition". Here, the VLL melts at $T_{m}$ into a coherent (disentangled) vortex liquid phase, where the free vortex lines are straight a well defined. In this phase, phase coher- ence along the field direction is still intact, and does not disappear before a new "entangled" phase transition at $T_{e}>T_{m}$. More recently and in this work, by lpoger simulation time on larger systems, it is found $17,22,13,102$ that $T_{e}=T_{m}$, and the VLL melts directly into the incoherent vortex liquid.

\section{B. Change in vortex-tangle connectivity}

We next discuss in some detail the results obtained for the quantity $O_{L}$, which probes the connectivity of the vortex-tangle in extreme type-II superconductors. We will make the following point: as for the zero field case, the increasingly sharp change in $O_{L}$ from zero to one in finite field, with increasing system size, also denotes a phase transition where a global $U(1)$-symmetry is broken. This refers to a $U(1)$-symmetry associated with the vortex-content of the Ginzburg-Landau theory. As argued in Section IIF, this symmetry of the vortex content of the theory is seen explicitly when rewriting it to a gauge-theory involving a local complex matterfield, see Section IIF. We also discuss the finite size effects of $O_{L}$, in systems with slab geometry, i.e. where $L_{x} / L_{z} \approx L_{y} / L_{z}>1$, as well as in cubic systems.

In Fig. 9 we show $O_{L}$ as a function of temperature for several system sizes, $\mathcal{V}=20^{3}, 60^{3}, 120^{3}$. We see that for increasing system sizes, the largest temperature where $O_{L}=0$ increases, while the smallest temperature where $O_{L}=1$ decreases.

This finite-size effect in the behavior of $O_{L}$ can not be explained by the $2 D$ non-relativistic boson-analogy picture. In this picture the transverse wandering $u(z)$ ff $^{-}$ vortex lines along the field direction $(\hat{z})$ is given by 25.47

$$
<(u(z)-u(0))^{2}>=2 D z ; \quad D=\frac{\Gamma^{2} k_{B} T}{\varepsilon} .
$$

We see in Eq. 34 that the probability of finding a vortex line with finite line-tension traversing the system in a direction perpendicular to the magnetic field, without using PBC along the field direction, should decrease with increasing system size. Thus, if the vortex liquid regime is always describable as a liquid of vortex lines, then an inescapable consequence of this picture would be that $T_{L}$ should shift to higher temperature with increasing system size. This is in clear contrast to the finite size effect of $O_{L}$ shown in Fig. 9. In the zero field case, $O_{L}=0$ indicates that the line tension of vortex loops is finite, while $O=1$ indicates that the line tension of vortex loops is zero. The temperature where $O_{L}$ jumps from zero to one is the critical temperature for the S-N phase transition. 

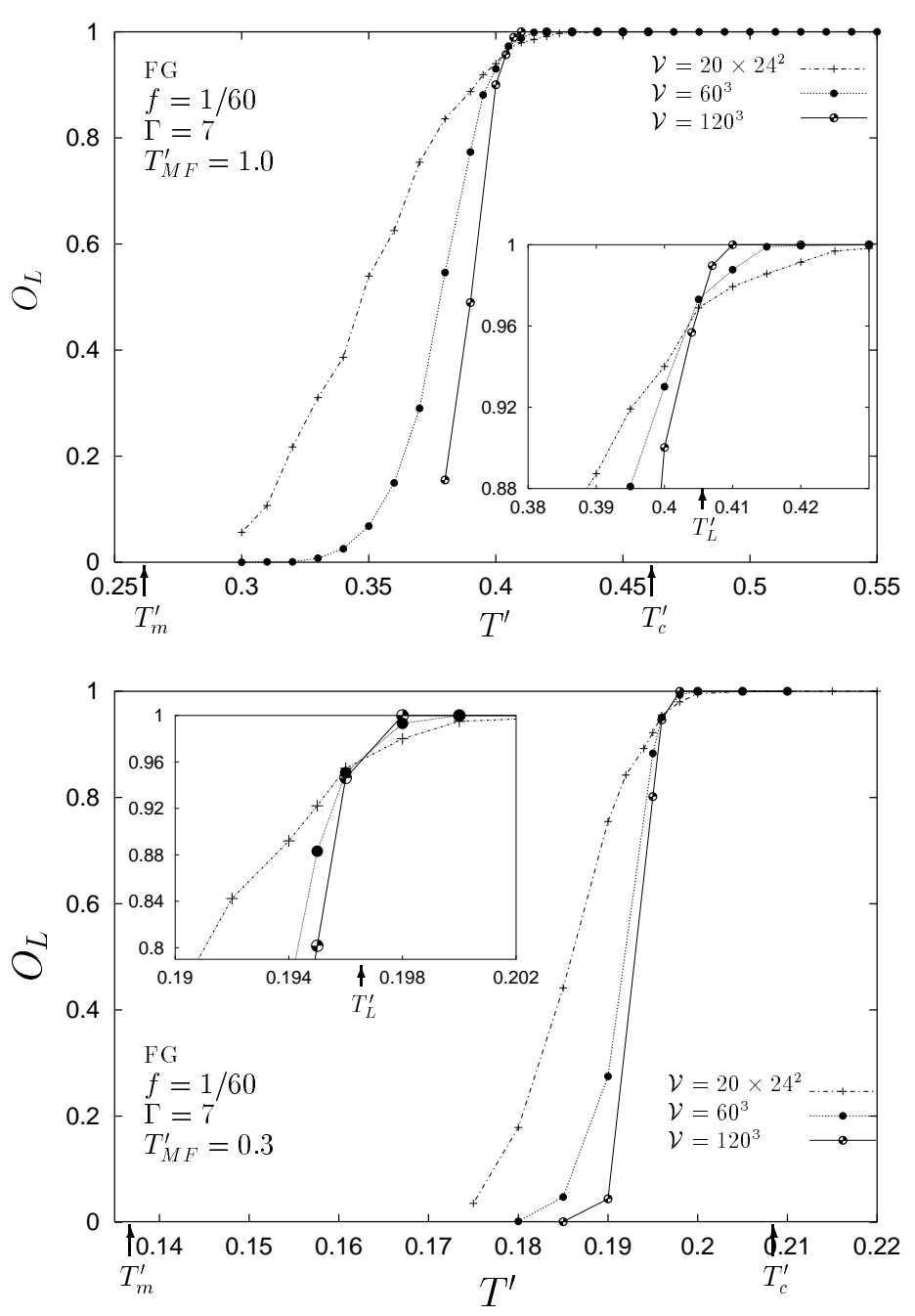

FIG. 9. Top panel: $O_{L}$ as a function of temperature for the Ginzburg-Landau model in a frozen gauge approximation with $f=1 / 60, \Gamma=7, T_{M F}^{\prime}=1, a_{\mu} / \xi_{\mu}=6$, and $\mathcal{V}=20^{3}, 60^{3}, 120^{3}$. For increasing system size the largest temperature where $O_{L}=0$ increases and the smallest temperature where $O_{L}=1$ decreases. Thus, in the thermodynamical limit, there exists a well defined temperature $T_{L}$ where $O_{L}$ jump sharply from zero to one. If we use the criteria $O_{L} \sim \bullet .9$, to determine $T_{L}$, we find that $T_{L}$ monotonically decreases to a limiting value for increasing system sizes. The inset shows the details of $O_{L}$ close to $T_{L}$. Note how the curves for $O_{L}$ all cross at the same temperature with increasing $L$. Note also how the lowest $T$ at which $O_{L}=1$ actually decreases with $L$. Bottom panel: Same as for top panel, but with $T_{M F}^{\prime}=0.3$.

We now focus on the inset of Fig. 9. Note how the curves for $O_{L}$ cross, and reach a value $O_{L}=1$ for progressively lower temperatures as $L$ increases. If a picture of the vortex-liquid in terms of well-defined vortex lines with non-zero linetension were applicable to this point, one would expect the point $T_{L}$ at which $O_{L}$ reaches the value 1 , to move monotonically up with $L$. The crossings of the curves for $O_{L}$ observed in the inset of Fig.
9 simply would not occur. Note also the similarity of this finite-size effect, and the ones observed in Fig. 圆 for the zero-field case. There, it was argued that such a finite-size effect was strongly indicative of a percolation threshold for thermally induced unbound vortex-loops in the thermodynamic limit 99 . The crossing point $T_{\text {cross }}$ seems a likely candidate for the limiting value of $T_{L}$ as $L \rightarrow \infty$, see Fig. 12 and the more detailed discussion below. This, in our view, provides strong numerical evidence that the progressively more abrupt change in the connectivity of the vortex-tangle as $L \rightarrow \infty$, is a real feature of the vortex system that survives in the thermodynamic limit, also at a finite magnetic field. In other words, the geometric transition signalled by the change in $O_{L}$ seems to be a real feature and not an artifact of small systems. Whether or not it also corresponds to a finite-field thermodynamic phase-transition will be discussed below.

In the vortex representation, Eq. 11 the $U(1)$ symmetry to be broken is hidden, and can only be explored implicitly using the conservation of $N_{\mu}$. The connection is made explicit by rewriting the vortex Hamiltonian in the disorder-field language, see Eq.12 of Section IIF. Below $T_{L}$, only field induced vortex line percolate the system. Thus, $N_{x}=N_{y}=0$ and $N_{z}=f L_{x} L_{y}$. Here, $f L_{x} L_{y}$ is the number of field induced vortex lines. For $T>T_{L}$, in addition to the field induced vortex lines, thermally excited "vortex lines" also exist. Thus, above $T_{L}, N_{\mu}$ is not a conserved quantity and the global $U(1)$ symmetry is broken, as for the zero field case.

In Ref. 13 it was claimed that because the longitudinal superfluid density vanished precisely at the melting line, as now found by several author 1214.96 including the isotropic case, the vortex lines could not be considered well defined in the vortex liquid phase. By itself, this is not a tenable conclusion. Nor does it follow automatically that the vortex-lines are entangled and that the mechanism for VLL melting is entanglement 49 . To substantiate such a claim one has to investigate in more detail the geometric properties of the vortex tangle in the liquid phase, as done above and in Refs. 43,14. Even if it should turn out that the loss of longitudinal superfluid density is entanglement it is probably more appropriate to view the entanglement as triggered by VLL melting transition rather than the converse. However, it is worth while pointing out at this stage that there is now consensus on the fact that at intermediate fields and above, the VLL melts into an incoherent vortex-liquid and that there does exist a regime where the molten phase consists of intact vortex lines, remarks to the contrary in Ref. 13 not withstanding. Moreover, various Monte-Carlo simulations agree that the Lindemanp-criterion for VLL melting applies in this regime $13,60.43,49$. In the low-field regime, far less consensus has so far been reached. Therefore, the question of whether vortex loops influence VLL melting or not, and whether there exists a genuine transition line $T_{L}(B)$ inside the vortex liquid, are two separate issues. 


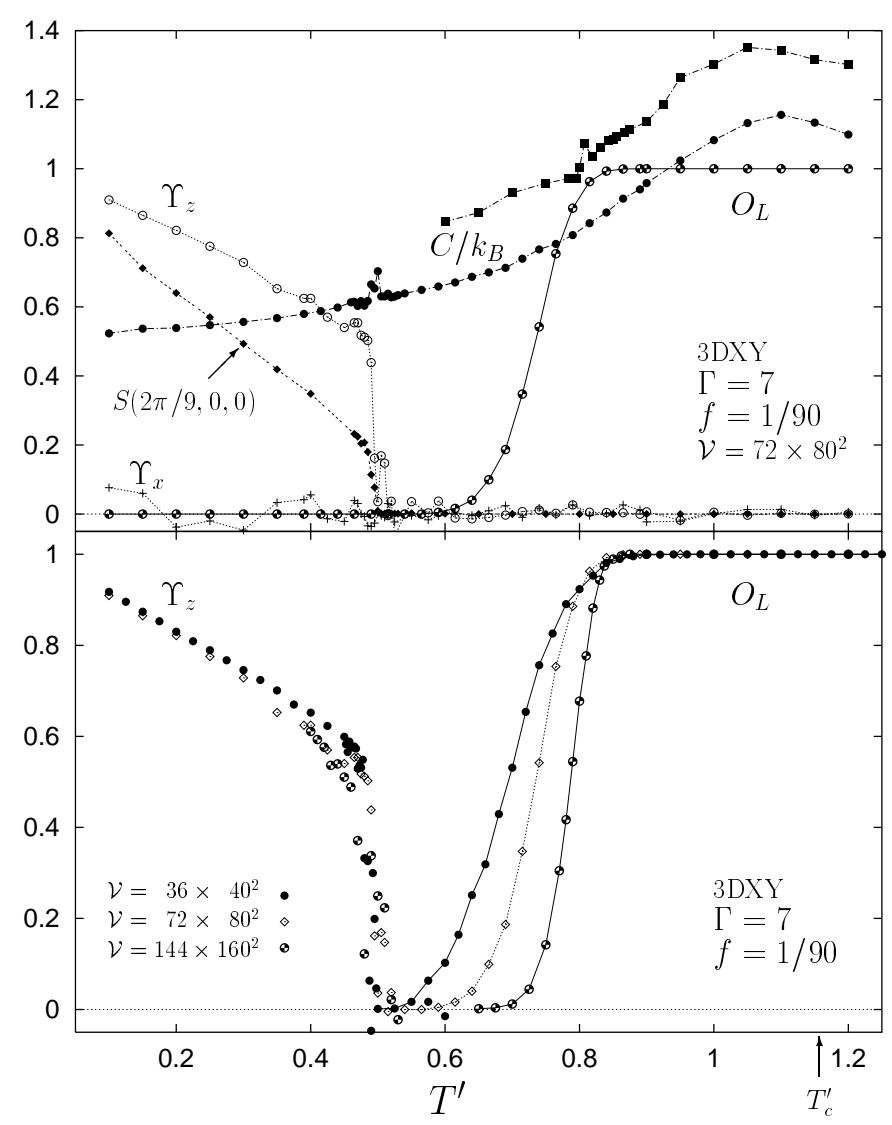

FIG. 10. Top panel: Helicity moduli $\Upsilon_{x}$ and $\Upsilon_{z}$, structure function $S\left(\mathbf{K}, k_{z}=0\right)$ and $O_{L}$ for the $3 D X Y$-model as a function of temperature for $f=1 / 90, \Gamma=7$, and system size $\mathcal{V}=72 \times 80 \times 80$

Bottom panel: $\Upsilon_{z}$ and $O_{L}$ for increasing system sizes. For increasing system size the largest temperature where $O_{L}=0$ increases and the smallest temperature where $O_{L}=1$ decreases. Thus, in the thermodynamical limit, there exists a well defined temperature $T_{L}$ where $O_{L}$ jumps sharply from zero to one, precisely as seen in the zero-field case. Shown is also specific heat for a system of size $\mathcal{V}=360^{3}$ (shifted up by $0.2 k_{B}$ for clarity).

We hereafter focus on simulation results obtained for the $3 D X Y$-model. There is no qualitative difference between the results for this model, and the GinzburgLandau model. In Fig. 10 we show results for the $3 D X Y$ model $f=1 / 90, \Gamma=7$. The top panel shows structure factor, superfluid density along the field, specific heat and $O_{L}$ for a system of size $72 \times 80 \times 80$. The bottom panel shows a sharpening of $O_{L}$ for increasing system sizes. The trend in the change in the vortex-tangle connectivity is precisely the same as that seen for $f=1 / 60$ within the Ginzburg-Landau model including amplitude fluctuations. The lowest temperature at which $O_{L}$ rises from zero, increases with system size, but the highest temperature at which it reaches the value $O_{L}=1$ decreases with system size. Again, we find a feature which indi- cates that a change in the vortex-tangle connectivity is undergoing a change.

\section{Effect of varying system aspect ratio}

According to the $2 D$ non-relativistic boson-analogy of the vortex-liquid, $T_{L}$ should be proportional to the aspect ratio $L_{x} / L_{z} 103$.
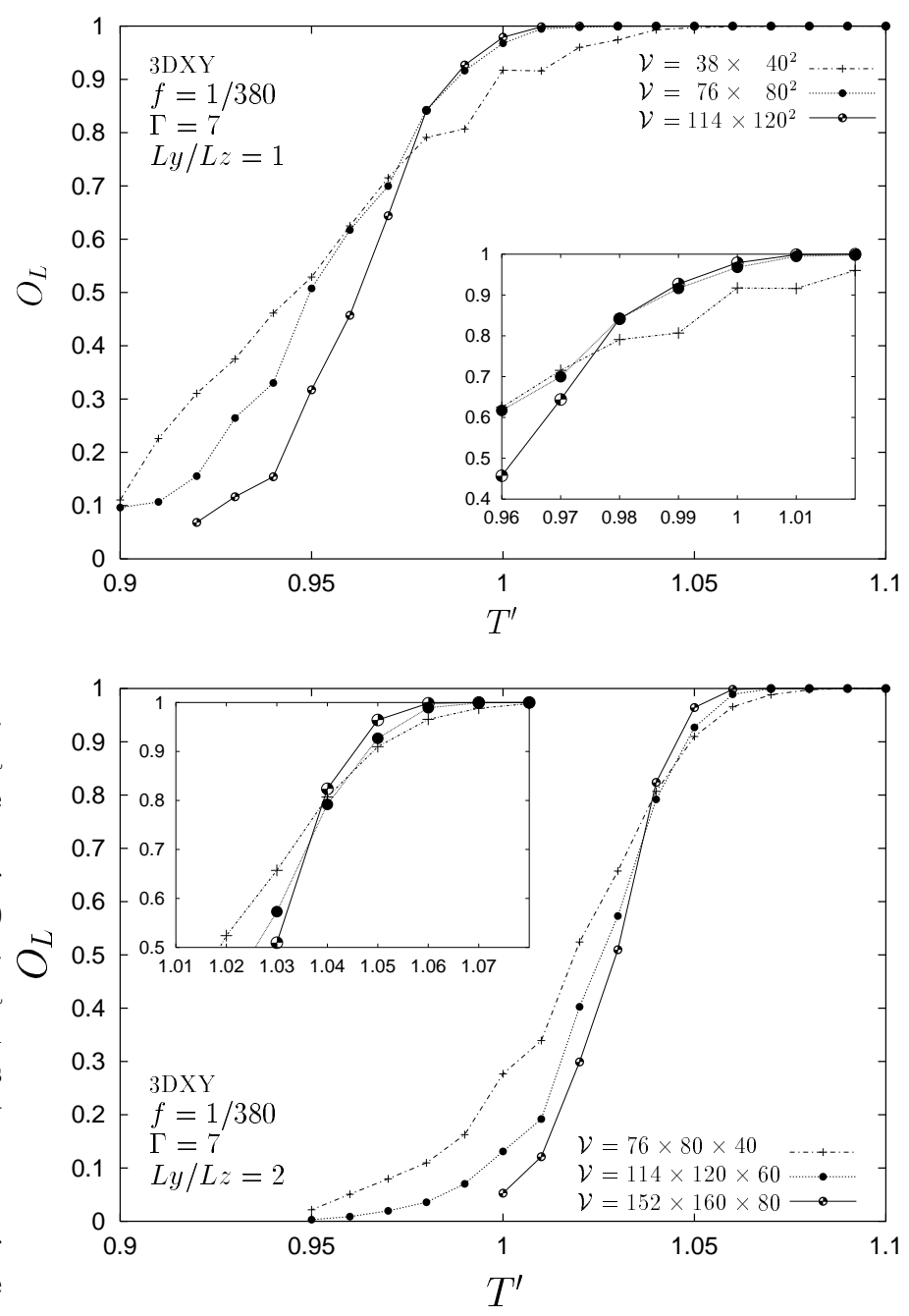

FIG. 11. $O_{L}$ as a function of system temperature, obtained within the $3 D X Y$-model for $f=1 / 380, \Gamma=7$, for various system sizes. Top panel: Aspect ratio $L_{y} / L_{z}=1$.

Bottom panel: aspect ratio $L_{y} / L_{z}=2$. Insets show details of the curve-crossings close to $T_{L}$. Note that while the linesonly approximation would predict a change in the crossing temperature of roughly a factor 2 , they only change by about $5 \%$, which is within the uncertainty of the estimate for the crossing temperature.

To further investigate the possibility of a breakdown of vortex-line physics inside the vortex-liquid regime, we 
consider the crossing feature found in $O_{L}$ in more detail for various aspect ratios $L_{x} / L_{z}$ of the systems on which the simulations are done. In this paragraph, we carry out the simulations using the $3 D X Y$-model with the parameters $f=1 / 380$ and $\Gamma=7$. We have varied the field to illustrate that the features of $O_{L}$ are the same as for the higher fields, but do become sharper. Furthermore, comparing the results obtained from the FG model to the results obtained from the $3 D X Y$-model, we again find that these models give qualitatively the same results when parameters are comparable.
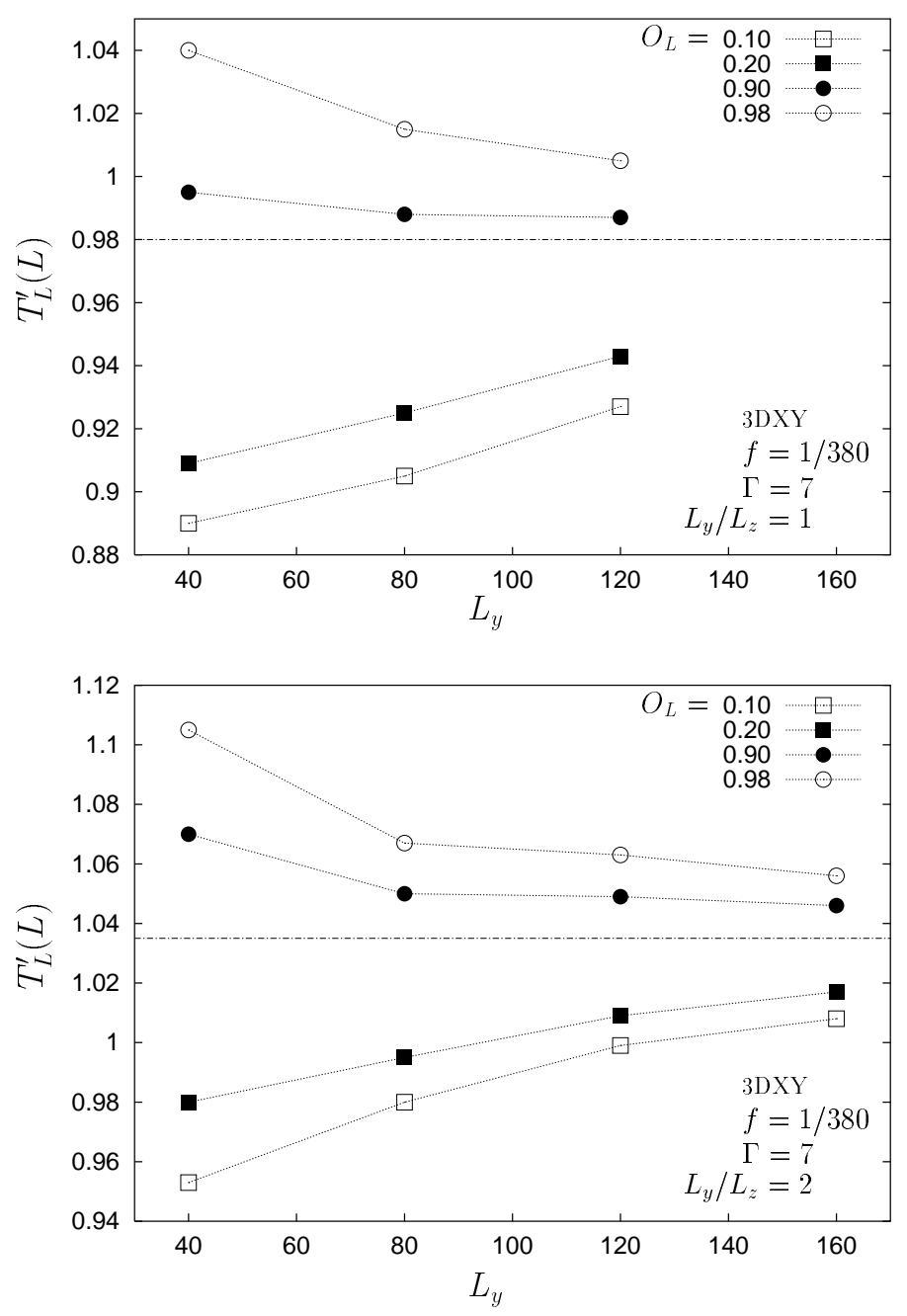

FIG. 12. Finite-size effect in $T_{L}$ as obtained for the $3 D X Y$-model using four sets of defining criteria for $T_{L}$, $O_{L}=(0.10,0.20,0.90,0.95)$. Each of the criteria gives converging curves for $T_{L}(L)$, whose limiting values are estimated by the crossing temperatures in the insets of Fig. 11. Top panel: aspect ratio $L_{y} / L_{z}=1$.

Bottom panel: aspect ratio $L_{y} / L_{z}=2$. Note that the limiting values for $L_{y} / L_{z}=1$ and 2 differ by about $5 \%$, whereas according to a vortex-line liquid picture, they should differ by about a factor of 2 .
In Fig. 11, we show $O_{L}$ as a function of $T$ for the $3 D X Y$-model with parameters $f=1 / 380, \Gamma=7$, for a system of size $L_{x} \sim L_{y}=L_{z}$ in one case, and $L_{x} \approx L_{y}$ and $L_{z}=0.5 L_{y}$ in the other case. Using the crossing temperature in the insets as an estimate for the temperature $T_{L}$ in the thermodynamic limit, as for the zero-field case, we see that this temperature changes very little when changing the aspect ratio by a factor of 2 . This indicates that in the thermodynamic limit there is only one $T_{L}$, regardless of the system shape. What these results indicate, is that the expectation one has based on the $2 \mathrm{D}$ non-relativistic bose-analogy of the vortex liquid, namely that $T_{L}$ should scale with $L_{x} / L_{z}$, is not borne out. Note that the present case is very different from the situation encountered in the $3 D$ Ising-model where a percolation threshold for overturned spins in an ordered spin state is found at a temperature which is lower than the critical temperature 8 .

This may be further illustrated by considering the finite-size effect of $T_{L}$, for two different aspect ratios $L_{x} / L_{z}=1$ and $L_{x} / L_{z}=2$. We investigate this by defining $T_{L}$ by four sets of criteria, namely the temperature at which $O_{L}=(0.10,0.20,0.90,0.95)$. If the curves for $O_{L}$ sharpen up, as seen in the above results, it is ultimately immaterial what sets of criteria are being used. The sets will give converging curves for $T_{L}(L)$, one coming up from below and one coming down from above, see Fig. 12. We may use the best estimate for the crossing temperatures in Fig. 11 as an estimate for what the limiting value of $T_{L}$ will be in the thermodynamic limit. These results illustrate two important points, namely i) $T_{L}$ does not move up monotonically with system size, but saturates at a specific value as $L \rightarrow \infty$ precisely as for the zero-field case, and ii) the limiting value of $T_{L}$ is independent of aspect ratio. Both of these two points contradict expectations based on a vortex-line liquid picture of the molten phase of the Abrikosov VLL.

\section{Scaling of the melting line $T_{m}(B)$}

In Fig. 13, we show data from various simulations, of the vortex lattice melting line $T_{m}(B)$. We want to emphasize the fact that there are two distinct scaling regimes for the melting line $T_{m}(B)$, one at high fields which somewhat arbitrarily denote high-field scaling regime 104, and one at low magnetic fields which we identify to be $3 D X Y$-scaling.

The dotted straight line is the curve given by 104 $k_{B} T_{m}(B) / J_{0}=0.41 y ; y^{2}<4$, where $y=1 / \sqrt{f} \Gamma$. It describes the published numerically obtained melting lines for large enough filling fractions $y^{2}<4$ or so well, in our case with $\Gamma=7$ corresponding to approximately $f>1 / 200$. On the other hand, for $y^{2}>4$, clear deviations from linear behavior is seen. 

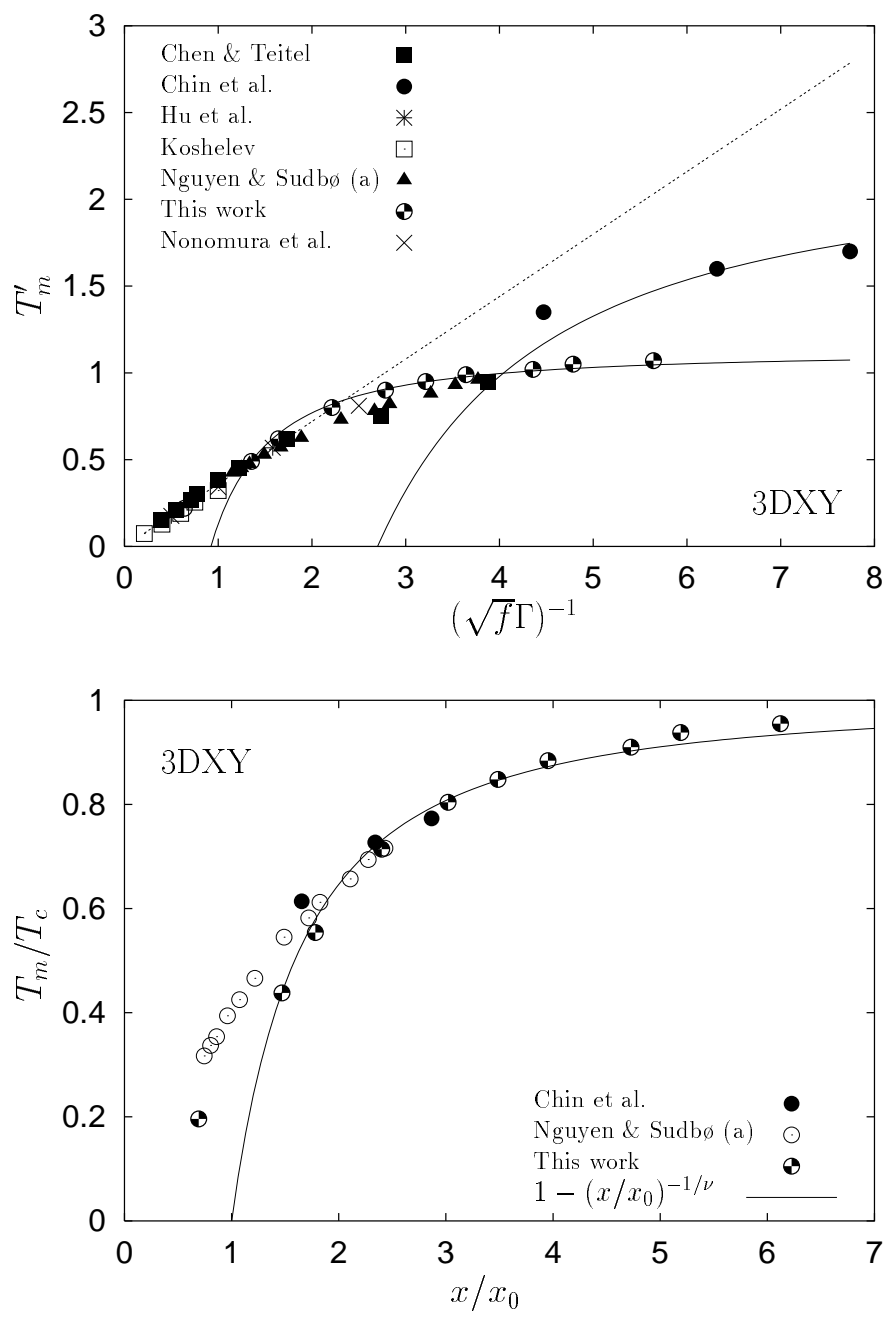

FIG. 13 Top panel: Melting temperature $T_{m}^{\prime}(B)$ of the vortex lattice as a function of $y=1 / \sqrt{f} \Gamma$. At large enough filling fractions, $y<2.0, T_{m}^{\prime}(B)$ obtained from various simulations on the $3 D X Y$-model and boson-analogues of the vortex system, agree and is well described by $T_{m}^{\prime}(B)=0.41 y$, the dotted line. At low filling fractions, $y>2.0$, there is a crossover to $3 D X Y$ critical-scaling of $T_{m}(B)$. The solid lines through the two sets of data points are $3 D X Y$ critical-scaling functions, described in the text.

Bottom panel: Normalized melting temperature $T_{m}^{\prime}(B) / T_{c}^{\prime}$ as a function of the varibale $x / x_{0}$, where $x=1 / \sqrt{f}$ and $x_{0}$ is an anisotropy dependent fitting parameter. Solid line is the $3 D X Y$ scaling function $h(x)=1-\left(x / x_{0}\right)^{-1 / \nu}$, where $\nu=0.67$.

The melting curves obtained for $\Gamma=1$ in Ref. 14 shown by the filled circles, and for $\Gamma=7$ in Ref. 13 shown by the half-filled circles saturate at low filling fractions $f$ to the values given by the zero-field critical temperature, $T_{c}$. For $\Gamma=1$, we have $k_{p} T_{c} / J_{0}=2.2$, while for $\Gamma=7$ we have $k_{\beta} T_{c} / J_{0}=1.1243$. The data given by the filled triangles 17 are obtained on the $3 D X Y$-model with an anisotropy parameter $\Gamma=3$. As $\Gamma$ increases from 1 , the zero-field transition temperature $T_{c}$ rapidly approaches its $2 D$ value, although the transition is always $3 D$ in character for finite anisotropy. Hence, the results from the anisotropic $3 D X Y$-model with $\Gamma=317$ are very close to those of the $3 D X Y$-model with $\Gamma=7$, see Ref. 13 .

The results of Ref. 105, obtained by fixing $f=1 / 15$ and varying $\Gamma \in(1, . .10)$, agree entirely with our results of Refs. 13, 14, 43 in the low-field regime $1 / \sqrt{f} \Gamma>2$. The significance of all these three sets of points is that they fall significantly below the straight line obtained from the high-field scaling of the melting line.

Note also that even if we normalize the melting line $T_{m}(\beta)$ quite arbitrarily, with a factor $1 /(1-$ $\left.T_{m} / T_{c}\right)^{2 \nu 104}$, this might take out the strong downward curvature of the data in the top panel of Fig. 13, but there is absolutely no reason for why the slope of the resulting curve in the low field regime, which would be a straight line, should be the same as in the high-field regime.

Assuming $3 D X Y$-scaling for the melting line when $y>>2$, i.e. $B /\left|1-T / T_{c}\right|^{2 \nu}=B_{0}$ where $B_{0}$ is a fieldscale that depends on anisotropy, we find $k_{B} T_{m}(B) / J_{0}=$ $\left(k_{B} T_{c} / J_{0}\right)\left[1-\left(x_{0} / \Gamma y\right)^{1 / \nu}\right]$ on the melting line, and where the last term is negligible for low fields. Hence, we find that the melting line saturates to the true critical temperature $T_{c}$, as it obviously must. The dotted straight line $k_{B} T_{m} / J_{0}=0.41 y$, overshoots $T_{c}$ as the field is lowered. The Monte-Carlo results follow this line at large fields, but are however starting to be arrested in their tracks by the zero-field vortex-loop critical fluctuations already at around $y=2$, thus crossing over to $3 D X Y$ critical scaling, as our Monte-Carlo simulations results show.

In the top panel of Fig. 13, we have drawn the function $T_{m}(B) / J_{0}=\left(T_{c} / J_{0}\right)\left[1-\left(x_{0} / y \Gamma\right)^{1 / \nu}\right]$ through the two sets of points obtained from Monte-Carlo simulations for $y>4$ and $\Gamma=1,7$, given by filled and half-filled circles, respectively. Using $x_{0}=2.70$ for $\Gamma=1$ and $x_{0}=6.45$ for $\Gamma=7$, we find that the $3 D X Y$ scaling function given above fits the Monte-Carlo data well for $y>4$, while the high-field scaling is excellent for $y<2$. Note how vastly different the scaling of $T_{m}(B)$ in the two regimes $y<2$ and $y>4$ is.

The bottom panel of Fig. 13 shows the low-field melting line $T_{m}(B)$ normalized by the zero-field critical temperature, obtained from simulations of the $3 D X Y$ model with $\Gamma=1, k_{B} T_{c} / J_{0}=2.2$ in Ref. 14, $\Gamma=3, k_{B} T_{c} / J_{0}=$ 1.34 in Ref. 13, and $\Gamma=7, k_{B} T_{c} / J_{0}=1.12$ in this work and in Ref. 43, plotted in terms of the variable $x / x_{0}$, where $x=1 / \sqrt{f}$ and $x_{0}$ is a fitting parameter for each $\Gamma$. The corresponding values of $\Gamma$ and $x_{0}$ are $(1,2.70),(3,4.65)$, and $(7,6.45)$. For $x / x_{0} \approx 2$ or less, i.e. at large enough fields, we see that deviations from $3 D X$-scaling occur. For $\Gamma=(1,3,7)$ this corresponds to $1 / f=(30,90,160)$, respectively. The line through the low-field data, is the $3 D X Y$ scaling function $1-\left(x / x_{0}\right)^{-1 / \nu}$. Notice the sharp bending of the $3 D X Y$ scaling function as $x / x_{0}$ increases beyond the value 3 , and how the available numerically obtained melting curves 
follow this line. This, in our view, provides strong numerical support for the notion that at low filling fractions $f \Gamma^{2}<<1$, the melting line $T_{m}(B)$ obeys $3 D X Y$ critical scaling, while it follows follows a quite different "meanfield" type of scaling $T_{m}(B) \sim 1 / \sqrt{B}$ at large fields 104 .

The field above which deviations from $3 D X Y$ critical scaling is seen, increases as the anisotropy goes down. This is due to the fact that with increasing $\Gamma$, the melting curve becomes flatter at low fields, leaving the critical region more rapidly as $\Gamma$ increases. The width of the critical region appears to widen only slightly with increasing anisotropy 13 , whereas the melting line rapidly becomes flatter at low fields, such that this is the dominant effect in determining the field at which the melting line enters the critical region.

\section{E. Phase diagram, clean limit}

A summary of all of the above is contained in Fig.14, we have included results from filling fractions $1 / f \in$ $[90, . .1560]$. The results we have obtained pertain to an extreme type-II superconductor in the absence of disorder, since we are primarily interested in the intrinsic properties of this phase-diagram excluding the severe complications due to disorder. There is a lowtemperature vortex-line lattice phase. When the vortex lattice melts, it melts directly into an incoherent vortex-liquid with zero longitudinal superfluid density. The transverse superfluid density has been eliminated at temperature far below those where the VLL melts, by choosing low enough filling fractions to eliminate an unwanted commensuration effect due to the presence of the numerical lattice on which the theory is defined.

At zero magnetic field, we have demonstrated that an alternative way of describing the superconductor-normal metal transition, in addition to the phase-disordering picture using the Ginzburg-Landau order parameter, is in terms of an unbinding of vortex-loops. We emphasize that although the quantity $O_{L}$ we have focused on is not an order parameter, it may be tied to an order via the discussion in Section IIF. By including amplitude fluctuations explicitly in the Ginzburg-Landau theory, it is shown that this vortex-loop unbinding does not lead to critical amplitude fluctuations. A generalized "stiffness" characterizing the low-temperature phase which vanishes at the transition, is the long-wavelength vortex-line tension $\varepsilon(T)$, or equivalently the free energy per unit length of the the thermally induced vortex loops of the system.

In a finite magnetic field, we find indications of a change in the vortex-tangle connectivity across the system at a temperature $T_{L}(B)$, whose zero-field endpoint is $T_{c}$. This has been done by monitoring the quantity $O_{L}$ in the Ginzburg-Landau theory or the $3 D X Y$-model in the same way as for the zero-field case. $O_{L}$ has precisely the same characteristica at finite fields and zero field. In the regime $T_{m}(B)<T<T_{L}(B)$, the connectivity across the system of the vortex-tangle of the molten phase is given entirely by the field induced vortex lines. This appears to change across the line $T_{L}(B)$. We have been able to tie $O_{L}$ to an order parameter even at finite field, see Section IIF, involving a breaking of a $U(1)$-symmetry across the line $T_{L}(B)$.

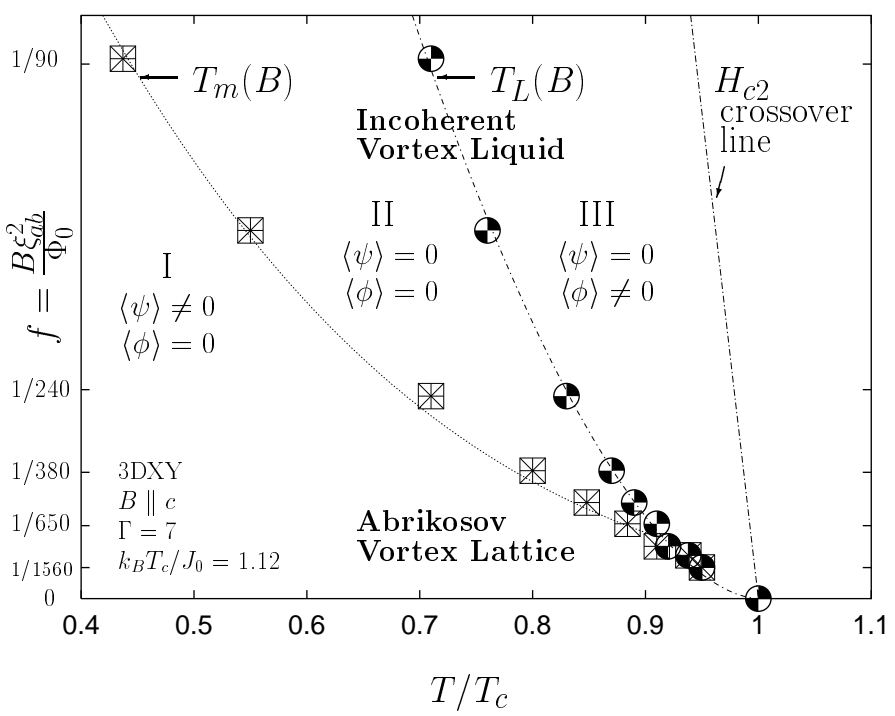

FIG. 14. $B-T$ phase-diagram for extreme type-II superconductors based on Monte-Carlo simulations of the $3 D X Y$ model with $B \| c$ and $\Gamma=7$. The phase-diagram splits into three differient regimes I, II, and III, characterized by the values of the Ginzburg-Landau and dual order parameters.

At low magnetic fields, to the accuracy of our calculations, we have found that the VLL melting line and the line $T_{L}(B)$ merge at low fields. Below these low magnetic fields, the picture of the molten phase as a vortex-line liquid appears to be questionable. For fields well above the point where $T_{m}(B)$ and $T_{L}(B)$ merge, we have found that the position of the VLL melting line is well described by a Lindemann criterion with Lindemann number $c \approx 0.25$, estimated from the Debye-Waller factor.

Note that the rewriting of the theory Eq. 11 to Eq. 12 is exact. The onset of the expectation value $\langle\phi\rangle$ takes place when vortex-loops unbind. Moreover, the theory Eq. 12 exhibits an explicit $U(1)$-symmetry. When this connection is made, it seems very reasonable to tie the observed change in the vortex-tangle connectivity to a vortex-loop unbinding and hence an onset of $\langle\phi\rangle$, i.e. the order parameter and the symmetry being broken in the transition, have been identified.

\section{SUMMARY AND DISCUSSION}

We have explored the $(B, T)$ phase diagram for extreme type II superconductors using two simplified ver- 
sions of the the Ginzburg-Landau model: i) The frozen gauge (FG) approximation where the gauge-field is fixed, while the phase and amplitude of the superconducting order parameter are allowed to fluctuate, and ii) the uniformly frustrated 3D XY model where only phase fluctuations are allowed for. The former is obviously a more general model than the latter, while the latter is a commonly accepted model in the studies of fluctuation effects in extreme type-II superconductors. Our results show that in the $\kappa \rightarrow \infty$-limit, where suppression of gaugefluctuations is an exact feature of a superconductor, amplitude fluctuations are completely dominated by phasefluctuations over a sizeable temperature regime. The local order field $\left\langle\psi^{\prime}(\mathbf{r})\right\rangle$, as well as the helicity modulus (global phase stiffness) $\Upsilon_{\mu}$ develop an expectation value for $T<T_{c}$, and explicitly break the usual $U(1)$-symmetry present in the Ginzburg-Landau theory. In contrast to this, the local Cooper-pair density $\left\langle\left|\psi^{\prime}\right|^{2}\right\rangle$, is finite both above and below $T_{c}$. Our precise calculations close to $T=T_{c}$ has brought out clearly its singular temperature derivative at $T=T_{c}$. Below, we list the main results of this paper.

$$
\underline{B}=\mathbf{0}
$$

- In zero field, we have shown that the superconductingnormal metal phase-transition is described by a vortexloop unbinding. This is achieved by correlating a detailed study of qualitative changes in the vortex-loop distribution function $D(p)$ with calculations of superfluid density, condensate density, specific heat, amplitude fluctuations, and change in vortex-tangle connectivity, both including and excluding amplitude fluctuations of the Ginzburg-Landau order-parameter. The topological phase-fluctuations destroying the superconducting phase-coherence are thus unambiguously identified as thermally induced vortex loops. When amplitude fluctuations are included explicitly, they are found to be far from critical. In other words, the vortex-loop unbinding may not be viewed as a reparametrization of critical amplitude fluctuations of the Ginzburg-Landau order parameter, as is sometimes claimed.

- The vortex-content of the Ginzburg-Landau theory, formulated in SectionIIE, is characterized by its own $U(1)$-symmetry which becomes explicit on a further $e x$ act reformulation of the vortex sector in terms of a new gauge-field, see Section IIF. The low-temperature phase of the vortex-sector of the Ginzburg-Landau theory, where all vortex-loops are confined, exhibits a $U(1)$ symmetry. This symmetry of the vortex sector reflects the fact that there is a number conservation of vortex loops extending across the entire superconductor. In zero magnetic field, the conserved number is zero, and the distribution function for closed vortex loops of perimeter $p$ is an exponential function with length scale given by $L_{0}(T)=k_{B} T / \varepsilon(T)$, where $\varepsilon(T)$ is the vortex-line tension. At the zero-field critical temperature, we find vortex-loops with an algebraic distribution of perimeters, concomitant with an abrupt change in the connectivity of the vortex tangle in extreme type-II superconductors.
The vortex line tension is found to vanish as a power law as $T_{c}$ is approached from below, $\varepsilon(T) \sim\left|T-T_{c}\right|^{\gamma}$, with $\gamma=1.45 \pm 0.05$.

- Both the change in the distribution function of closed vortex loops, and the abrupt change in the connectivity of the vortex tangle, shows that there is a diverging length in the problem, i.e. $L_{0}(T) \rightarrow \infty ; T \rightarrow T_{c}^{-}$. At this point, the number of closed vortex-loops extending through the system is no longer a conserved number equal to zero, it becomes finite and undergoes thermal fluctuations. Therefore, the $U(1)$-symmetry characterizing the low-temperature vortex-phase is broken, the vortexsystem has suffered a vortex-loop blowout, or unbinding.

- The connection between the power-law behavior close to $T_{c}$ of the vortex-line tension and the anomalous dimension $\eta_{\phi}$ of the dual field $\phi$ was discussed in Section IV C. Relating the power-law for the vortex-line tension to the susceptibility exponent $\gamma$ of the $\phi$-field, in conjunction with the Fisher scaling law $\gamma=\nu\left(2-\eta_{\phi}\right)$, allowed us to extract the value $\eta_{\phi}=-0.18 \mp 0.07$. This result was compared to renormalization group calculation performed directly on the dual theory, for which the vortex-loop unbinding is the phase-transition, and excellent agreement was found. Note the negative sign of $\eta_{\phi}$ in the extreme type-II case.

$\mathbf{B} \neq \mathbf{0}$

- In finite field, we have studied the phase-diagram over a wide range of filling fractions $f$, corresponding to $1 / f \in[20, \ldots, 1560]$. Only a subset is shown explicitly, but all results are summarized in Fig. 14. The VLL is found to melt in a first order phase transition, for all filling fractions considered, into a completely incoherent vortex liquid characterized by zero global phase coherence in all directions. At intermediate fields, the VLL melts into a liquid of vortex lines, whose position in the $(B, T)$-phase diagram is well estimated by the Lindemann criterion with a Lindemann number $\approx 0.25$.

- We have performed a scaling analysis for the melting line for all filling fractions considered. We find a crossover from mean-field type scaling at elevated fields to $3 D X Y$-scaling behavior at small fields, showing that for the aniosotropies we have considered, the melting line of the vortex-lattice at low fields is significantly affected by zero-field critical fluctuations in a sizeable region of the phase-diagram.

- Significantly, in addition to the VLL melting transition line $T_{m}(B)$, we find indications of another transition line, $T_{L}(B)$, inside the vortex liquid. This line is the finite-field extension of the zero-field vortex-loop unbinding, and has an endpoint which is the zero-field critical temperature $T_{c}$. Below $T_{L}$, connectivity of the vortexsystem is determined exclusively by the field-induced vortex lines. All vortex-lines threading the entire system are field-induced. Above $T_{L}$, this changes, as discussed in Section IVB. Above $T_{L}(B)$ there exist vortex lines that thread the systen also perpendicular to the magnetic field, without using periodic boundary conditions along the $z$-axis. 
- We have performed a large-scale study of the finitesize effects in $T_{L}$, and found that the temperature where the vortex-tangle connectivity changes does not move up with system size, like it would have done in a vortexline liquid. In the $2 D$ non-relativistic boson-analogy of vortex-liquid, such vortex-configurations are never found. The symmetry broken at $T_{L}$ is a global $U(1)$ symmetry, associated with the number-conservation of vortex paths threading the entire system, the considerations are similar to the zero-field case. In a finite magnetic field, this symmetry is hidden in the usual Ginzburg-Landau local order field representation, but is brought out by a dual description of the Ginzburg-Landau theory. In zero field, $T_{L}$ and $T_{c}$ are identical and there is only one phase transition.

- We have found that the vortex-system in the clean limit appears to be able to exhibit three distinct phases, I, II, and III shown in Fig. 14, characterized by the values of the Ginzburg-Landau order parameter $\langle\psi\rangle$ and its dual order-parameter $\langle\phi\rangle$. Here, we explicitly utilized the connection of Section IIE between the vortex-tangle connectivity probe $O_{L}$ and the $U(1)$-ordering in the dual field $\phi$. We found the three regimes

$$
\begin{array}{cc}
\text { Region I }:<\psi>\neq 0 & <\phi>=0, \\
\text { Region II }:<\psi>=0 & <\phi>=0, \\
\text { Region III }:<\psi>=0 & <\phi>\neq 0 .
\end{array}
$$

At low fields, we have found that region II vanishes. Note that the transition-line $T_{L}(B)$ separating the regions II and III inside the vortex-liquid, was brought out solely through the dual description, it could not have been detected by studying the Ginzburg-Landau order parameter $\langle\psi\rangle$, or any local function of it.

A few further comments are in order. In the lowfield regime, within a lines-only picture of the molten phase, one finds that the longitudinal correlation length of field-induced vortex lines above melting increases, due to the increased distance between field induced lines, being given by

$$
\xi_{z}=\frac{1}{\Gamma^{2}} \sqrt{\frac{\Phi_{0}}{B}} .
$$

It was therefore pointed out in Ref. 60 that in order to correctly predict the direct transition from the Abrikosov vortex lattice to a phase-incoherent vortex liquid at low magnetic fields, or equivalently predict the direct transition from the crystal phase to the superfluid phase of $2 D$ non-relativistic bosons at $T=0$ at low magnetic, sufficiently large systems in the $z$-direction must be used. The use of too small systems could result in observing, merely as a result of a finite-size effect, a normal $T=0$ $2 D$ non-relativic bose-fluid, or equivalently a disentangled vortex-liquid. The former cannot exist in the thermodynamic limit in the absence of disorder, on quite general grounds.

The above is a valid point of concern within the $2 D$ boson-liquid analogy of the vortex system when looking for entanglement. It is no longer a point of concern if the lines-only approximation is abandoned and the connectivity of the vortex tangle is probed rather than entanglement. (Precisely how to establish a criterion for when field-induced vortex lines are entangled, also appears to be problematic to say the least). For all fields we have considered, and for all sample geometries we have used, it is clear from our results that we have been able to correctly predict the direct transition from the Abrikosov vortex lattice to the phase-incoherent vortex liquid. The onset of $O_{L}$ and the change in the vortextangle connectivity is a separate matter. The vortexconfigurations dominating the contribution to a change in $O_{L}$, are thermally induced unbound vortex loops and not field-induced flux lines. Our results in the low-field regime are therefore not artifacts of considering too small systems in the $z$-direction. Quite the contrary, since we see the change in $O_{L}$ also when making the system flatter, it supports the proposition that there exists a regime in the $(B, T)$ phase-diagram, beyond the line $T_{L}(B)$, where the notion of a vortex-line liquid physics most probably should be revised.

The $U(1)$-transition line $T_{L}(B)$ has the zero-field superfluid normal state transition $T_{c}$ as an endpoint. It is a feature of extreme type-II superconductors, even homogenous, isotropic three-dimensional ones, and should moreover occur in Helium ${ }^{4}$ which is a perfectly threedimensional, homogenous, isotropic superfluid. The proposed transition therefore is not in any obvious way connected to various previously proposed quite intriguing scenarios leading to a loss of local line-tension of field induced_yortices, often referred to as "decouplingtransitions"106.107. These phenomena rely on the layeredness of the superconducting compounds, however they have no symmetry-breaking or order parameter associated with them, but most importantly do not have a zerofield counterpart. Moreover, probing phase-coherence between adjacent layers in a layered superconductor as was for instance done in Ref.106 (see their Eq. 28) probes maximum $q_{z}$-behavior, a part of reciprocal space not usually associated with critical phenomena, which are infrared singularities. Probing phase-coherence between increasingly distant layers ultimately amounts to computing the helicity modulus $\Upsilon_{z}$, which cannot vanish above the melting-line of the vortex lattice in the thermodynamic limit, in the absence of disorder.

The $T_{L}(B)$ line is potentially an important line in the $(B, T)$ phase diagram. It locates the position in the $(B, T)$-diagram where the line-only approximation of the vortex-liquid breaks down. Pinning of vortices by extended objects such as columnar pins may very well turn out to be inefficient beyond the line $T_{L}(B)$. It also shows that the line-only approximation can be used to describe the vortex-liquid phase and the first order melting transition of the VLL at $T_{M}(B)$ only for large and intermediate magnetic induction. In low magnetic fields, on the other hand, $T_{L}(B)$ and $T_{m}(B)$ collapse into a single 
line 43 . Here, it would appear that a line-only approximation does not describe the vortex liquid properly. The fields where the line only approximation fails in the entire liquic_regime are expected to be of order $1 T$ or less in $\mathrm{YBCO} 43$.

\section{ACKNOWLEDGMENTS}

Support from the Research Council of Norway (Norges Forskningsråd) under Grants No. 110566/410, No. $110569 / 410$, as well as a grant for computing time under the Program for Super-computing, is gratefully acknowledged. We thank E. W. Carlson, S. K. Chin, J. Hove, D. A. Huse, J. S. Høye, S. A. Kivelson, J. M. Kosterlitz, F. Ravndal, A. M. J. Schakel, and N. C. Yeh for useful communications. In particular, we thank Z. Tešanović and P. B. Weichman for discussions and critical readings of the manuscript. Finally, we would like to express our sincere thanks to Jørn Amundsen for his invaluable and continuing assistance in optimizing our computer codes for use on the CrayT3E.

${ }^{1}$ A. A. Abrikosov, Zh. Eksp. Teor. Fiz. 32, 1442 (1957).

${ }^{2}$ We use the abbreviation VLL rather than FLL (flux-line lattice) throughout this paper. The reason is that while flux lines, i.e. lines of confined magnetic flux, do not exist in the extreme type-II limit $\lambda \rightarrow \infty$, vortex lines do.

${ }^{3}$ G. Eilenberger, Phys. Rev. 153, 584 (1967).

${ }^{4}$ P. L. Gammel et al., Phys. Rev. Lett. 59, 2592 (1987).

${ }^{5}$ D. R. Nelson, Phys. Rev. Lett. 60, 1973 (1988).

${ }^{6}$ A. Houghton, R. A. Pelcovits, and A. Sudbø, Phys. Rev B 40, 6763 (1989).

${ }^{7}$ E. H. Brandt, J. Low. Temp. Phys. 26, 709 (1977).

${ }^{8}$ E. H. Brandt, J. Low. Temp. Phys. 26, 735 (1977).

${ }^{9}$ H. Safar et al., Phys. Rev. Lett. 69, 824 (1992).

${ }^{10}$ E. Brezin, D. R. Nelson, and A. Thiaville, Phys. Rev. B 31, 7124 (1985).

${ }^{11}$ R. E. Hetzel, A. Sudb $\varnothing$, and D. A. Huse, Phys. Rev. Lett. 69, 518 (1992).

${ }^{12}$ X. Hu, S. Miyashita, and M. Tachiki, Phys. Rev. Lett. 79, 3498 (1997).

${ }^{13}$ A. K. Nguyen and A. Sudbø, Phys. Rev. B 58, 2802 (1998).

${ }^{14}$ S. Chin, A. K. Nguyen, and A. Sudbø, Phys. Rev. B 59, 14017 (1999).

${ }^{15}$ Z. Tešanović, Phys. Rev. B 51, 16204 (1995).

${ }^{16}$ A. K. Nguyen, A. Sudbø, and R. E. Hetzel, Phys. Rev. Lett. 77, 1592 (1996).

${ }^{17}$ A. K. Nguyen and A. Sudbø, Phys. Rev. B 57, 3123 (1998).

18 Z. Tešanović, Phys. Rev. B 59, 6449 (1999).

${ }^{19}$ V. J. Emery and S. A. Kivelson, Nature 374, 434 (1995).
${ }^{20}$ V. J. Emery and S. A. Kivelson, Journal of Physics and Chemistry of Solids 59, 1705 (1999).

${ }^{21}$ V. J. Emery and S. A. Kivelson, condmat-9902179, and references therein. In addition, the role of classical phasefluctuations in high- $T_{c}$ cuprates have recently been considered using Monte-Carlo simulations of the 3DXY-model, i.e. phase-only approximation to the full Ginzburg-Landau theory, see Ref. 22 .

${ }^{22}$ E. W. Carlson, S. A. Kivelson, V. J. Emery, and E. Manousakis, condmat-9902077.

${ }^{23}$ A. Griffin, Excitations in a Bose-condensed liquid (Cambridge university press, Cambridge, 1993).

${ }^{24}$ P. G. de Gennes and J. Prost, The physics of Liquid Crystals, 2 ed. (Clarendon Press, Oxford, 1993).

${ }^{25} \mathrm{H}$. Kleinert, Gauge fields in condensed matter (World Scientific, Singapore, 1989), Vol. 1.

${ }^{26}$ K. Farakos, K. Kajantie, K. Rummukainen, and M. Shaposhnikov, Nucl. Phys. B 425, 67 (1994).

${ }^{27}$ K. Kajantie, M. Karjalainen, M. Laine, and J. Peisa, Nucl. Phys. B 520, 345 (1998).

${ }^{28}$ M. B. Hindmarsh and T. W. B. Kibble, Rep. Prog. Phys. 58, 477 (1995).

${ }^{29}$ W. H. Zurek, Phys. Rept. 276, 177 (1996).

${ }^{30}$ A. Vilenkin and E. P. S. Shellard, Cosmic strings and other topological defects (Cambridge University Press, Cambridge, 1994).

${ }^{31}$ C. Dasgupta and B. I. Halperin, Phys. Rev. Lett. 47, 1556 (1981).

32 G. A. Williams, Phys. Rev. Lett. 59, 1926 (1987).

${ }^{33}$ G. A. Williams, Physica B 165, 769 (1990).

${ }^{34}$ S. R. Shenoy, Phys. Rev. B 40, 5056 (1989).

${ }^{35}$ S. R. Shenoy, Phys. Rev. Lett. 72, 400 (1994).

${ }^{36}$ G. A. Williams, Phys. Rev. Lett. 82, 1201 (1999).

${ }^{37}$ B. I. Halperin, Les Houches Proceedings 1980 (Ed. R. Balian, North Holland, Amsterdam, 1980).

${ }^{38}$ M. B. Salamon and J. Shi, Phys. Rev. B 47, 5520 (1993).

39 A. Junod et al., Physica C 275, 245 (1997).

${ }^{40}$ M. Roulin, A. Junod, and E. Walker, Physica C 282, 1401 (1997).

${ }^{41}$ M. Roulin, Thesis Nr. 2989: Specific heat of the high-T superconductor $\mathrm{YBa}_{2} \mathrm{Cu}_{3} \mathrm{O}_{x}$ near $T_{c}$, versus Oxygen consentration (Université de Genevè, Genevè, 1998).

${ }^{42}$ M. Roulin, A. Junod, A. Erb, and E. Walker, Phys. Rev. Lett. 80, 1722 (1998).

${ }^{43}$ A. K. Nguyen and A. Sudbø, EuroPhys. Lett. xx, yyy (1999).

${ }^{44}$ N. D. Antunes, L. M. A. Bettencourt, and M. Hindmarsh, Phys. Rev. Lett. 80, 908 (1998).

${ }^{45}$ N. D. Antunes and L. M. A. Bettencourt, Phys. Rev. Lett. 81, 3083 (1998).

${ }^{46}$ G. Blatter et al., Rev. Mod. Phys. 66, 1125 (1994).

${ }^{47} \mathrm{H}$. Nordborg, Vortices and 2D bosons: A path-integral Monte Carlo study (ETH, Zürich, 1997).

${ }^{48}$ Ref. 6 explicitly avoids a discussion of the low-field part of the melting curve. The calculation was performed from the low-temperature side using a Lindemann-criterion for melting, and the fluctuation propagator for the vortex system was obtained for the case where magnetic fields surrounding vortices strongly overlap, however with $\lambda$ being finite. At very low fields where the distance between 
field-induced vortex lines becomes large, the Lindemann criterion most likely becomes unphysical, and is therefore not useful for locating the VLL melting transition line as $B \rightarrow 0, T \rightarrow T_{c}$.

${ }^{49}$ Y. Nonomura, X. Hu, and M. Tachiki, cond-mat/9812002

${ }^{50}$ V. L. Ginzburg and L. D. Landau, Zh. Eksp. Teor. Fiz. 20, 1064 (1950).

${ }^{51}$ K. Takanaka, Phys. Stat. Sol. B 68, 623 (1975).

${ }^{52}$ W. E. Lawrence and S. Doniach, in Proc. 12th Int. Conf. Low Temp. Phys. LT12, edited by E. Kanda (Academic Press of Japan, Kyoto, 1971), p. 361.

${ }^{53}$ A. M. Polyakov, Phys. Lett. 59B, 82 (1975).

${ }^{54}$ Y.-H. Li and S. Teitel, Phys. Rev. Lett. 66, 3301 (1991).

${ }^{55} \mathrm{M}$. Tinkham, Introduction to Superconductivity, 2 ed. (McGraw-Hill, New York, USA, 1996).

${ }^{56}$ M. Friesen and P. Muzikar, Physica C 302, 67 (1998).

${ }^{57}$ In most cases involving interacting field-theories exhibiting certain symmetries in statistical mechanics, a symmetry exhibited by the theory will be broken under some conditions, unless it is prohibited on fundamental grounds. For the three-dimensional dual field-theory presented here, no such grounds appear to exist.

58 M. Kiometzis, H. Kleinert, and A. M. J. Schakel, Prog. of Phys. 43, 697 (1995).

${ }^{59}$ M. J. W. Dodgson, V. B. Geshkenbein, H. Nordborg, and G. Blatter, Phys. Rev. Lett. 80, 837 (1998).

${ }^{60}$ H. Nordborg and G. Blatter, Phys. Rev. B 58, 14556 (1998).

61 J. Hu and A. H. MacDonald, Phys. Rev. B 56, 2788 (1997).

62 M. Roulin, A. Junod, A. Erb, and E. Walker, J. Low. Temp. Phys. 105, 1099 (1996).

${ }^{63}$ M. Roulin, A. Junod, and E. Walker, Science 273, 1210 (1996).

${ }^{64}$ M. E. Fisher, M. N. Barber, and D. Jasnow, Phys. Rev. A 8, 1111 (1973).

${ }^{65}$ Y.-H. Li and S. Teitel, Phys. Rev. B 47, 359 (1993).

66 B. D. Josephson, Proc. Phys. Soc. 92, 269 (1967).

${ }^{67}$ M. E. Fisher, Phys. Rev. 180, 594 (1969).

${ }^{68}$ G. F. Rushbrooke, J. Chem. Phys. 39, 842 (1963).

${ }^{69}$ See W. Janke, Phys. Lett., A 148, 306 (1990); M. Hasenbusch and A. P. Gottlob, Physica A 201, 593 (1993); P. Butera and M. Comi, Phys, Rev. B 56, 8212 (1997); R. Guida and J. Zinn-Justin, J. Phys. A 31, 8103 (1998). The most recent and accurate result for $\eta$, appears to be given by M. Hasenbusch and T. Török, condmat-9904408, who claim $\eta=0.0381 \pm 0.0002$, obtained directly via high-precision Monte-Carlo simulations, and without using scaling-laws.

${ }^{70}$ We thank P. B. Weichman for useful communications on this point.

${ }^{71}$ Y.-H. Li and S. Teitel, Phys. Rev. B 49, 4136 (1994).

72 J. S. Høye and G. Stell, J. Stat. Phys. 77, 361 (1994).

${ }^{73}$ In Refs. 71, 17 a slightly simpler form $D(p) \sim$ $\exp \left(-\varepsilon(T) p / k_{B} T\right)$ was used. Adding an algebraic prefactor $p^{-\alpha}$ gives a better description of the numerical data over a wider temperature range.

74 This value of the exponent is based on a mapping of the vortex-loop gas to a $3 D$ non-interacting non-relativistic bose-system 72 . It evidently gives a good fit to our numerical data. The fact that the bose-system is non-interacting reflects the fact that the vortices are well screened. This can only happen with infinite vortex-loops present, so the value $\alpha=5 / 2$ is expected to be a very precise value for $\alpha$ strictly speaking only immediately above $T_{c}$. Our simulation results are consistent with $\alpha=2.4 \pm 0.10$, if one attempts a more precise determination of $\alpha$ at $T=T_{c}$. This is essentially consistent with the results of Antunes and Bettencourt, who find $\alpha=2.23 \pm 0.04$, see Ref. 45 . It is possible that these authors have a more precise way of obtaining $\alpha$ than what Monte-Carlo methods offer. A very precise determination of $\alpha$ is however not our main concern in this paper, rather we wish to point out the fact that $D(p)$ clearly changes from exponential to power-law behavior. A simple scaling argument gives the too large value $\alpha=3$ which provides a moderately good fit to numerical data, see Ref. 17. However as noted in Ref. 36, the value $\alpha=2.4$ fits the numerical data for $D(p)$ in Ref. 17 better. The reason for this is that the scaling argument assumes that the vortex-loop perimeter scales with its diameter. These perimeters are, however, "crinkled" objects reducing the value of $\alpha$ from 3 to $\approx 2.5$.

${ }^{75}$ D. R. Nelson and H. S. Seung, Phys. Rev. B 39, 9153 (1989).

${ }^{76}$ E. A. Jagla and C. A. Balseiro, Phys. Rev. B. 53, 538 (1996).

77 The authors of Ref. 76 concluded that percolation was necessary in order to destroy the longitudinal superfluid density regardless of magnetic field, not unlike our own previous claim of Ref. 13. However, as the present work and the results of Ref. 43 show, the loss of longitudinal superfluid density does not necessarily imply that the connectivity of the vortex tangle changes dramatically. The loss of superfluid density always occurs at the VLL melting transition, which at elevated magnetic fields is well below the temperature where we find a change in connectivity. We believe, however, that the remarks of Refs. 76,13 are essentially correct at low magnetic fields. Namely, at low fields the VLL melting transition, loss of longitudinal superfluid density, and change of vortex-tangle connectivity occur at the same temperature.

${ }^{78} O_{L}$ measures the possibility of having percolation of connected vortex paths across the system in any direction, including directions perpendicular to any applied magnetic field. It is known from the $3 D$ Ising-model that one can measure percolation of overturned spins, finding a percolation threshold temperature $T_{p}$ that has nothing to do with the critical point $T_{c}$, in fact $T_{p}<T_{c}$. This is easily understood because the topological defects that destroy the spin-stiffness of the $3 D$ Ising-model are surfaces of overturned spins, a domain wall. On the other hand, percolation simply probes when a line of overturned spins appears. It costs less energy creating a line than a surface, and hence in the $3 D$ Ising-case, $T_{p}<T_{c}$. However, in the present case of the $F G / 3 D X Y$-model, $O_{L}$ probes the minimum condictions under which a vortex line connecting the system in a non-field direction can appear. More precisely, it measures the connectivity of the vortex tangle in the $3 D \mathrm{XY}$-model. The topological defects de- 
stroying the phase-stiffness of the $F G / 3 D X Y$-model are in fact line-defects in the form of closed vortex-loops. This is why $O_{L}$ can be tied to the onset of a local dual order parameter $\langle\phi\rangle$ (disorder parameter) and thus a symmetry breaking in the case of the $3 D \mathrm{XY}$-model, as in Section IIF, while the percolation threshold in the $3 D$ Ising model cannot.

${ }^{79}$ R. Cavalcanti, G. Carneiro, and A. Gartner, Europhys. Lett. 17, 449 (1992).

${ }^{80}$ M. Plischke and B. Bergersen, Equilibrium statistical physics, 2 ed. (World Scientific, Singapore, 1994).

${ }^{81}$ T. Chui and M. R. Giri, Phys. Lett. A 128, 49 (1988).

${ }^{82}$ W. Janke and T. Matsui, Phys. Rev. B 42, 10673 (1990).

${ }^{83}$ L. Onsager, in The collected works of Lars Onsager, edited by P. C. Hemmer, H. Holden, and S. K. Ratkje (World Scientific, Singapore, 1996), p. 1070.

${ }^{84}$ L. Onsager, Nuovo Cimento Suppl. 6, 249 (1949).

${ }^{85}$ R. P. Feynman, in Progress in Low Temperature Physics, edited by C. J. Gorter (North Holland, Amsterdam, 1955), Vol. 1, p. 17.

${ }^{86}$ W. N. Hardy et al., Proceedings of the 10th Anniversary HTS Workshop in Physics, Materials and Applications (World Scientific, Singapore, 1996).

${ }^{87}$ We thank S. A. Kivelson for a useful discussion on this point.

88 T. Chen and S. Teitel, Phys. Rev. B 55, 15197 (1997).

89 J. H. Akao, Phys. Rev. B 53, 6048 (1996).

${ }^{90} \mathrm{D}$. Stauffer and A. Aharony, Introduction to percolation theory (Taylor and Francis, London, 1994).

91 This is quite distinct from the loops that were invoked in M. P. A. Fisher, D. S. Fisher, and D. A. Huse, Phys. Rev. B 43, 130 (1991), where vortex half-loops were used to model flux-creep in a vortex-system. Introducing vortex half-loops, which necessarily must be attached to field- induced vortex-lines, always amounts to considering fluctuations of field induced quantities only.

${ }^{92}$ I. F. Herbut and Z. Tešanović, Phys. Rev. Lett. 764588 (1996); ibid, 78, 980 (1997); I. D. Lawrie, Phys. Rev. Lett., 78, 979 (1997).

${ }^{93}$ P. Olsson and S. Teitel, Phys. Rev. Lett. 80, 1964 (1998).

${ }^{94} \mathrm{~J}$. Hove and A. Sudbø, in preparation .

${ }^{95}$ We thank Z. Tešanović for many useful discussions on this point.

${ }^{96}$ P. Olsson and S. Teitel, Phys. Rev. Lett. 82, 2183 (1999).

${ }^{97}$ R. Cubitt et al., Nature 365, 407 (1993).

98 A. Oral et al., Phys. Rev. Lett. 80, 3610 (1998).

${ }^{99}$ H. Enriquez, Y. D. Wilde, and N. Bontemps, condmat/9801233.

${ }^{100}$ S. Ryu and D. Stroud, Phys. Rev. B 57, 14476 (1998).

101 T. Chen and S. Teitel, Phys. Rev. Lett. 74, 2792 (1995).

102 A. E. Koshelev, Phys. Rev B 56, 11201 (1997).

${ }^{103}$ H. Nordborg, private communication.

104 A. E. Koshelev and H. Nordborg, Phys. Rev B 59, 4358 (1999).

105 T. Chen and S. Teitel, Phys. Rev. B 55, 11766 (1997).

${ }^{106}$ L. I. Glazman and A. E. Koshelev, Phys. Rev. B43, 2835 (1991). In addition, it would also appear from Section IVB of the work of these authors, that what they investigate is partly a phenomenon taking place inside the vortexlattice phase. See also E. Frey, D. R. Nelson, and D. S. Fisher, Phys. Rev. B49, 9723 (1994). At low fields, the crossover-line established is inside the vortex liquid, and thus the proposed crossover diagram has a topology which is different than the calculated phase-diagram of our Fig. 14.

${ }^{107}$ L. N. Bulaevskii, M. Ledvij, and V. G. Kogan, Phys. Rev. Lett. 68, 3773 (1992). 